\title{
Bifurcation in a Differential-Algebra Predator-Prey System with Time Lag Effects
}

\author{
Wei Liu ${ }^{1,2}$ and Yaolin Jiang ${ }^{1, *}$ \\ ${ }^{1}$ School of Mathematics and Statistics, Xi'an Jiaotong University, Xi'an 710049, \\ China. \\ ${ }^{2}$ School of Mathematics and Computer Science, Xinyu University, Xinyu 338004, \\ China.
}

Received 7 March 2018; Accepted (in revised version) 7 May 2018.

\begin{abstract}
A predator-prey system with Holling type II functional response and a time lag is described by a delayed differential-algebra system and the local asymptotic stability and Hopf bifurcation of such system is studied. It is shown that if the time lag increases, a sequence of Hopf bifurcations can occur. The stability and direction of the Hopf bifurcations are studied by using center manifold theory for functional differential equations. A numerical example illustrates our theoretical findings.
\end{abstract}

AMS subject classifications: 92D25

Key words: Predator-prey, bifurcation, time lag, parametrisation, harvesting.

\section{Introduction}

The interactions between predators and preys are arguably the building blocks of complex ecosystems [37]. Therefore, since the last century the dynamical behaviors of predatorprey systems attracted extensive attention in theoretical ecology and applied mathematics. The functional response is a significant factor in population dynamics. It shows the average number of prey per time unit for an individual predator. The simplest functional response $m x(t)$, where $m>0$ is the capture rate of the predator species, is a linear function of the prey density $x(t)$, introduced in the celebrated Lotka-Volterra predator-prey system [37]. It is clear that the linear functional response is unbounded and does not produce saturation. Later on, a more reasonable Holling type II functional response of the form of $m x(t) /(a+x(t))$ with the so-called half capturing saturation constant $a>0$ has been considered $[19,20]$. The Holling type II functional response is bounded. It indicates that the amount of prey consumed by a predator per unit of time is still finite, even if the prey density $x(t)$ is very large. In the present paper, we consider the basic predator-prey system of the Holling type II - viz.

*Corresponding author. Email addresses: wliu2015@163.com (W. Liu), yljiang@xjtu.edu.cn (Y.L. Jiang) 


$$
\begin{aligned}
& \dot{x}(t)=x(t)\left(r-\frac{r}{K} x(t)-\frac{m y(t)}{a+x(t)}\right), \\
& \dot{y}(t)=y(t)\left(-d+\frac{c m x(t)}{a+x(t)}\right),
\end{aligned}
$$

where $x(t)$ and $y(t)$ denote, respectively, the population densities of prey and predators at the time $t$. The positive constants $r, K, d$ and $c$ represent, respectively, the intrinsic growth rate of prey population in absence of predators, the carrying capacity of the environment, the death rate of predator species and the conversion rate of predator population (converting the captured prey into predators).

It was pointed out by Kuang [26] that any population dynamics of a predator-prey system without time lag is an approximation at best, since the time lag reflects the past history of the system and can affect the present and future dynamical behaviors. On the other hand, compared to ordinary differential systems, the differential systems with time lag usually exhibit much richer and more complex dynamics such as stability switches, multistability, Takens-Bogdanov bifurcation, oscillations and periodic motions - cf. Refs. [14, $26,29,33,40,42,43,45,49,50]$. Following May [33], we add a time lag $\tau$ to the specific prey growth term $r-(r / K) x(t)$ in the system (1.1). Here, $\tau$ can be considered as the gestation period of prey species. Consequently, the predator-prey system with a time lag takes the form

$$
\begin{aligned}
& \dot{x}(t)=x(t)\left(r-\frac{r}{K} x(t-\tau)-\frac{m y(t)}{a+x(t)}\right), \\
& \dot{y}(t)=y(t)\left(-d+\frac{c m x(t)}{a+x(t)}\right) .
\end{aligned}
$$

Let us note that harvesting on biological populations are often practiced in real life, and the catches are commonly sold in the market for economic benefits. Therefore, here we consider a system with prey harvesting and investigate the economic benefits of the harvesting by using an economic equation in Ref. [15] — viz.

$$
\text { Net Economic Revenue }=\text { Total Revenue }- \text { Total Cost. }
$$

Let $E(t)$ denote the harvesting effort on the prey species, $\mathbb{P}(t)$ the unit selling price of the catch and $\mathbb{C}(t)$ the unit harvesting cost. Then $E(t) x(t)$ is the number of catches. We assume that the market has a constant demand for the catches, so that they always can be sold out here. Note that the selling price $\mathbb{P}(t)$ is a decreasing function of the market supply $E(t) x(t)[12,31]$, and the harvesting cost $\mathbb{C}(t)$ varies inversely to the population density $x(t)$. Consequently, we take $\mathbb{P}(t)$ and $\mathbb{C}(t)$ as $b /(c+E(t) x(t))$ and $e / x(t)$ respectively, where $b, c$ and $e$ are positive constants, $b / c$ is the maximum unit selling price and $e$ the harvesting cost for the unit population density of prey species. Thus, taking into account the economic equation (1.3), we have

$$
\begin{aligned}
& \text { Total Revenue }=\mathbb{P}(t) E(t) x(t)=\frac{b E(t) x(t)}{c+E(t) x(t)}, \\
& \text { Total Cost }=\mathbb{C}(t) E(t) x(t)=e E(t) .
\end{aligned}
$$


It follows that the net profit $v$ of the harvesting is

$$
v=E(t) x(t)\left(\frac{b}{c+E(t) x(t)}-\frac{e}{x(t)}\right) .
$$

Incorporating the Eq. (1.4) into the system (1.2), we arrive at a delayed differential-algebra equations for predator-prey model with time lag — viz.

$$
\begin{aligned}
& \dot{x}(t)=x(t)\left(r-\frac{r}{K} x(t-\tau)-\frac{m y(t)}{a+x(t)}-E(t)\right), \\
& \dot{y}(t)=y(t)\left(-d+\frac{c m x(t)}{a+x(t)}\right), \\
& 0=E(t) x(t)\left(\frac{b}{c+E(t) x(t)}-\frac{e}{x(t)}\right)-v,
\end{aligned}
$$

where $E(t)$ is considered as a variable in what follows.

In this study, the differential-algebra predator-prey system (1.5) is supplemented with the initial conditions

$$
x(\theta)>0, \quad \theta \in[-\tau, 0], \quad y(0)>0, \quad E(0)>0,
$$

where $x(\theta) \in C([-\tau, 0], \mathbb{R})$ is a continuous bounded function, $C([-\tau, 0], \mathbb{R})$ represents a Banach space of continuous mappings from the interval $[-\tau, 0]$ into the set of real numbers $\mathbb{R}$.

Nowadays, most of the predator-prey systems with harvesting are modelled by differential equations - cf. Refs. [6, 24, 27, 36, 46] and the references therein. On the other hand, the harvested predator-prey system (1.5) considered here and also in Refs. [5, 28$30,39,47,48]$, are differential-algebra system, where economic benefits of the harvesting on biological population are taken into account. This is an important distinction from the common harvested predator-prey systems and it is more difficult to analyse the dynamics of such systems than the systems of differential equations. In particular, the stability and Hopf bifurcation of a delayed predator-prey system with the functional response $\sqrt{x(t)}$ and harvesting was studied in Ref. [29]. In addition, Zhang et al. [47] investigated the local stability and the possible bifurcations of a Leslie-Gower predator-prey system with predator harvesting and delays (maturation delay of predators and digestion delay of preys) by using the normal form method [13]. The existence of Hopf bifurcation and stability of bifurcating periodic solutions of an ordinary predator-prey system with the Holling type IV response function has been considered in [30], where Hopf bifurcation theorem [16] and the formal series method [44] have been used. Moreover, a number of discrete-time predator-prey systems, based on Poincaré and the forward Euler schemes have been developed and studied in $[5,39,48]$. Motivated by the constrained power systems presented in $[1,32,38]$, Li et al. [28] explored the singularity induced bifurcation phenomenon in an ordinary preydependent predator-prey system with nonlinear prey harvesting.

In this work, we discuss the population dynamics of the differential-algebra predatorprey system (1.5) in presence of time lag $\tau$ due to the gestation of prey population. 
In order to study the stability of positive equilibrium and bifurcating periodic orbits of the system (1.5), we use the stability switches results $[10,17]$ and the center manifold reduction method [18]. We also improved the predator-prey models from [5,28-30,39,47,48], where the case of constant unit selling price and unit harvesting cost was considered. Here they are time-varying variables, depending on the economics principles and harvesting cost. We note that this study, complement and extend previous works [5, 6, 24, 27-30,36, 39, 46-48].

\section{Local Asymptotic Stability and Hopf Bifurcations}

In this section, the local asymptotic stability of the differential-algebra predator-prey system (1.5) and the appearance of the Hopf bifurcations are considered. For this, we study the characteristic equation of a linearized system for (1.5) at its positive equilibrium. Let us recall the basic properties of the system (1.5).

Lemma 2.1. The solutions of the differential-algebra predator-prey system (1.5) with the initial values (1.6) and $v>0$ are positive for all $t>0$.

Proof. It follows from the first equation in (1.5) that

$$
\frac{\mathrm{d} x(t)}{x(t)}=\left(r-\frac{r}{K} x(t-\tau)-\frac{m y(t)}{a+x(t)}-E(t)\right) d t .
$$

Integrating this equation over interval $(0, t)$, we obtain that

$$
x(t)=x(0) \exp \left\{\int_{0}^{t}\left(r-\frac{r}{K} x(s-\tau)-\frac{m y(s)}{a+x(s)}-E(s)\right) d s\right\}>0
$$

for all $t>0$. Similar procedure applied to the second equation in (1.5) yields

$$
\frac{\mathrm{d} y(t)}{y(t)}=\left(-d+\frac{c m x(t)}{a+x(t)}\right) d t
$$

and, consequently,

$$
y(t)=y(0) \exp \left\{\int_{0}^{t}\left(-d+\frac{c m x(s)}{a+x(s)}\right) d s\right\}>0
$$

for all $t>0$. According to the definition of $E(t)$, this term is non-negative. Moreover, assuming that $E(t)=0$ for a number $t>0$, we obtain from the third equation in (1.5) that $v=0$, which contradicts the conditions of Lemma 2.1 .

Lemma 2.1 indicates that the trajectories of the differential-algebra predator-prey system (1.5) with the initial condition (1.6) and with $v>0$ belong to the set

$$
\mathbb{R}_{+}^{3}=\{(x(t), y(t), E(t)) \mid x(t)>0, y(t)>0, E(t)>0\} .
$$

Therefore, our next task is to guarantee the existence of the positive equilibrium of this system, since it is located in the first quadrant of $\mathbb{R}_{+}^{3}$. 
Assuming that $X_{0}=\left(x_{0}, y_{0}, E_{0}\right)^{T}$ is the equilibrium of the system (1.5), we conclude that the components of $X_{0}$ satisfy the equations

$$
\begin{aligned}
& r-\frac{r}{K} x_{0}-\frac{m y_{0}}{a+x_{0}}-E_{0}=0, \\
& -d+\frac{c m x_{0}}{a+x_{0}}=0, \\
& \frac{b E_{0} x_{0}}{c+E_{0} x_{0}}-e E_{0}-v=0 .
\end{aligned}
$$

The solutions of these equations are

$$
X_{0}=\left(x_{0}, y_{0}, E_{0}\right)^{T}=\left(x_{0}, \frac{a+x_{0}}{m}\left(r-\frac{r}{K} x_{0}-E_{0}\right), E_{0}\right)^{T},
$$

where $x_{0}=a d /(c m-d)$ and $E_{0}$ is the root of the quadratic equation $e x_{0} E_{0}^{2}+\left(c e+v x_{0}-\right.$ $\left.b x_{0}\right) E_{0}+c v=0$. Furthermore, since the equilibrium $X_{0}$ is positive, its components $x_{0}, y_{0}$ and $E_{0}$ are also positive. Therefore, here and in what follows, we always assume that

$$
c m>d, \quad b x_{0}>v x_{0}+c e, \quad\left(c e+v x_{0}-b x_{0}\right)^{2} \geq 4 c e v x_{0}, \quad r>\frac{r}{K} x_{0}+E_{0} .
$$

According to the qualitative theory of differential-algebra systems [21], the system (1.5) is locally equivalent to the following DAE system around its positive equilibrium $X_{0}$ :

$$
\begin{aligned}
& \dot{x}(t)=x(t)\left(r-\frac{r}{K} x(t-\tau)-\frac{m y(t)}{a+x(t)}-E(t)\right), \\
& \dot{y}(t)=y(t)\left(-d+\frac{c m x(t)}{a+x(t)}\right), \\
& \dot{E}(t)=f_{3}(x(t), y(t), E(t)), \\
& 0=E(t) x(t)\left(\frac{b}{c+E(t) x(t)}-\frac{e}{x(t)}\right)-v,
\end{aligned}
$$

where $f_{3}(x, y, E)$ is a continuous differentiable function such that $f_{3}\left(x_{0}, y_{0}, E_{0}\right)=0$. Note that explicit expression of $f_{3}(x, y, E)$ is not needed - cf. the Eqs. (2.6) below.

Set

$$
\begin{aligned}
& f(X):=\left(\begin{array}{c}
f_{1}(X) \\
f_{2}(X) \\
f_{3}(X)
\end{array}\right)=\left(\begin{array}{c}
x(t)\left(r-\frac{r}{K} x(t-\tau)-\frac{m y(t)}{a+x(t)}-E(t)\right) \\
y(t)\left(-d+\frac{c m x(t)}{a+x(t)}\right) \\
f_{3}(x(t), y(t), E(t))
\end{array}\right), \\
& g(X):=E(t) x(t)\left(\frac{b}{c+E(t) x(t)}-\frac{e}{x(t)}\right)-v, X=(x, y, E)^{T},
\end{aligned}
$$

and let $D$ denote the differential operator. Taking into account the Eqs. (2.2), we write

$$
D_{X} g\left(X_{0}\right)=\left(b c E_{0} /\left(c+E_{0} x_{0}\right)^{2}, 0,\left[b c x_{0}-e\left(c+E_{0} x_{0}\right)^{2}\right] /\left(c+E_{0} x_{0}\right)^{2}\right),
$$


Bifurcation in a Differential-Algebra Predator-Prey System with Time Lag Effects

so that rank $D_{X} g\left(X_{0}\right)=2$. Thus this system does not satisfy the conditions of the parametrisation method introduced in the appendix below. Therefore, in order to employ that method here, we consider the nonsingular transformation $X=Q \bar{X}$ of the system (2.2), where

$$
Q=\left(\begin{array}{lll}
1 & 0 & 0 \\
0 & 1 & 0 \\
R & 0 & 1
\end{array}\right), \quad R=-\frac{b c E_{0}}{b c x_{0}-e\left(c+E_{0} x_{0}\right)^{2}}, \quad \bar{X}(t)=(x(t), y(t), \bar{E}(t))^{T} .
$$

Then, this system takes the form

$$
\begin{aligned}
& \dot{x}(t)=x(t)\left(r-\frac{r}{K} x(t-\tau)-\frac{m y(t)}{a+x(t)}-(\bar{E}(t)+R x(t))\right), \\
& \dot{y}(t)=y(t)\left(-d+\frac{c m x(t)}{a+x(t)}\right) \\
& \dot{\bar{E}}(t)=f_{3}(x(t), y(t), \bar{E}(t)) \\
& 0=(\bar{E}(t)+R x(t)) x(t)\left(\frac{b}{c+(\bar{E}(t)+R x(t)) x(t)}-\frac{e}{x(t)}\right)-v,
\end{aligned}
$$

where $f_{3}(x, y, \bar{E})$ is a continuous differentiable function such that $f_{3}\left(\bar{X}_{0}\right)=0$ and

$$
\bar{X}_{0}=\left(x_{0}, y_{0}, \bar{E}_{0}\right)^{T}=\left(x_{0}, y_{0}, E_{0}+b c x_{0} E_{0} /\left[b c x_{0}-e\left(c+E_{0} x_{0}\right)^{2}\right]\right)^{T}
$$

is the equilibrium of the transformed DAE system (2.3). We again point out that an explicit expression for the function $f_{3}(x, y, \bar{E})$ is not needed for what follows - cf. the Eqs. (2.6).

We now write

$$
\begin{aligned}
& f(\bar{X})=\left(\begin{array}{l}
f_{1}(\bar{X}) \\
f_{2}(\bar{X}) \\
f_{3}(\bar{X})
\end{array}\right)=\left(\begin{array}{c}
x(t)\left(r-\frac{r}{K} x(t-\tau)-\frac{m y(t)}{a+x(t)}-(\bar{E}(t)+R x(t))\right) \\
y(t)\left(-d+\frac{c m x(t)}{a+x(t)}\right) \\
f_{3}(x(t), y(t), \bar{E}(t))
\end{array}\right), \\
& g(\bar{X})=(\bar{E}(t)+R x(t)) x(t)\left(\frac{b}{c+(\bar{E}(t)+R x(t)) x(t)}-\frac{e}{x(t)}\right)-v
\end{aligned}
$$

and since $\bar{E}_{0}=E_{0}-R x_{0}, R=-b c E_{0} /\left[b c x_{0}-e\left(c+E_{0} x_{0}\right)^{2}\right]$, then the system (2.3) takes the form

$$
\begin{aligned}
D_{\bar{X}} g\left(\bar{X}_{0}\right) & =\left(\frac{b c\left(E_{0}+R x_{0}\right)-e R\left(c+E_{0} x_{0}\right)^{2}}{\left(c+E_{0} x_{0}\right)^{2}}, 0, \frac{b c x_{0}-e\left(c+E_{0} x_{0}\right)^{2}}{\left(c+E_{0} x_{0}\right)^{2}}\right) \\
& =\left(0,0, \frac{b c x_{0}-e\left(c+E_{0} x_{0}\right)^{2}}{\left(c+E_{0} x_{0}\right)^{2}}\right),
\end{aligned}
$$

so that $\operatorname{rank} D_{\bar{X}} g\left(\bar{X}_{0}\right)=1$. Moreover, the transformed DAE system (2.3) satisfies the other condition of the parametrisation method in Appendix. 
According to Refs. [3,7], $g(\bar{X}(t)): \mathbb{R}^{3} \rightarrow \mathbb{R}$ is a continuous differentiable function, and since rank $D_{\bar{X}} g(\bar{X}(t))=1$, the regular solution set of $g(\bar{X}(t))$ defines 2-dimensional smooth manifold $\mathscr{M}_{g}=\left\{\bar{X}(t) \in \mathbb{R}^{3}: g(\bar{X}(t))=0\right\}$. We now consider the following parametrisation $\psi$ of $\mathscr{M}_{g}:$ For any $\bar{X}(t) \in B\left(\bar{X}_{0}\right) \subset \mathscr{M}_{g}$, let $Y(t)=\left(y_{1}(t), y_{2}(t)\right)^{T} \in \mathscr{N}_{\psi} \subset \mathbb{R}^{2}$ be a function such that

$$
\bar{X}(t)=\psi(Y(t))=\bar{X}_{0}+U_{0} Y(t)+V_{0} h(Y(t)) \text { and } \quad g(\psi(Y(t)))=0,
$$

where $B\left(\bar{X}_{0}\right)$ is an open neighborhood of $\bar{X}_{0}, \mathscr{N}_{\psi}=\psi^{-1}\left(B\left(\bar{X}_{0}\right)\right), h(Y(t)): \mathbb{R}^{2} \rightarrow \mathbb{R}$ a smooth mapping and

$$
U_{0}=\left(\begin{array}{ll}
1 & 0 \\
0 & 1 \\
0 & 0
\end{array}\right), \quad V_{0}=\left(\begin{array}{l}
0 \\
0 \\
1
\end{array}\right)
$$

Since $\mathscr{M}_{g}$ is a smooth manifold, the existence of $h$ follows from the implicit function theorem. Thus, the DAE system (2.3) can be now reduced to the parameterised system

$$
\dot{Y}(t)=U_{0}^{T} D_{\bar{X}} f\left(\bar{X}_{0}\right)\left(\begin{array}{c}
D_{\bar{X}} g\left(\bar{X}_{0}\right) \\
U_{0}^{T}
\end{array}\right)^{-1}\left(\begin{array}{c}
0 \\
I_{2}
\end{array}\right) Y(t)+o(|Y|) .
$$

For more details concerning the Eq. (2.5), the reader is referred to the Appendix below. In particular, it suggests that the parametric procedure is a version of the implicit function theorem. Hence, the zeros of the corresponding function can be derived locally, without finding global inverses.

Let us study the local stability of the equilibrium $\bar{X}_{0}$ of the equivalent DAE system (2.3). Since $\bar{E}_{0}=E_{0}-R x_{0}$, the Jacobi matrix of the linearised system of (2.3) evaluated at the equilibrium $\bar{X}_{0}$ has the form

$$
\begin{aligned}
& U_{0}^{T} D_{\bar{X}} f\left(\bar{X}_{0}\right)\left(\begin{array}{c}
D_{\bar{X}} g\left(\bar{X}_{0}\right) \\
U_{0}^{T}
\end{array}\right)^{-1}\left(\begin{array}{c}
0 \\
I_{2}
\end{array}\right)=\left(\begin{array}{c}
D_{\bar{X}} f_{1}\left(\bar{X}_{0}\right) \\
D_{\bar{X}} f_{2}\left(\bar{X}_{0}\right)
\end{array}\right)\left(\begin{array}{c}
D_{\bar{X}} g\left(\bar{X}_{0}\right) \\
U_{0}^{T}
\end{array}\right)^{-1}\left(\begin{array}{c}
0 \\
I_{2}
\end{array}\right) \\
& =\left(\begin{array}{ccc}
-\frac{r}{K} x_{0} e^{-\lambda \tau}+\frac{m x_{0} y_{0}}{\left(a+x_{0}\right)^{2}}-R x_{0} & -\frac{m x_{0}}{a+x_{0}} & -x_{0} \\
\frac{a c m y_{0}}{\left(a+x_{0}\right)^{2}} & 0 & 0
\end{array}\right)\left(\begin{array}{ccc}
0 & 0 & \frac{b c x_{0}-e\left(c+E_{0} x_{0}\right)^{2}}{\left(c+E_{0} x_{0}\right)^{2}} \\
1 & 0 & 0 \\
0 & 1 & 0
\end{array}\right)^{-1} \\
& \times\left(\begin{array}{ll}
0 & 0 \\
1 & 0 \\
0 & 1
\end{array}\right)=\left(\begin{array}{cc}
-\frac{r}{K} x_{0} e^{-\lambda \tau}+\frac{m x_{0} y_{0}}{\left(a+x_{0}\right)^{2}}-R x_{0} & -\frac{m x_{0}}{a+x_{0}} \\
\frac{a c m y_{0}}{\left(a+x_{0}\right)^{2}} & 0
\end{array}\right) .
\end{aligned}
$$

It is worth noting that $U_{0}^{T} D_{\bar{X}} f\left(\bar{X}_{0}\right)=\left(D_{\bar{X}} f_{1}\left(\bar{X}_{0}\right), D_{\bar{X}} f_{2}\left(\bar{X}_{0}\right)\right)^{T}$. Hence, explicit expressions for the terms $f_{3}(x, y, E)$ and $f_{3}(x, y, \bar{E})$ are not required.

In order to study the local asymptotic stability of the positive equilibrium $X_{0}$, we have to find the roots of the characteristic equation

$$
\lambda^{2}+\left(\frac{r}{K} x_{0} e^{-\lambda \tau}-\frac{m x_{0} y_{0}}{\left(a+x_{0}\right)^{2}}+R x_{0}\right) \lambda+\frac{a c m^{2} x_{0} y_{0}}{\left(a+x_{0}\right)^{3}}=0
$$


Bifurcation in a Differential-Algebra Predator-Prey System with Time Lag Effects

of the Jacobi matrix (2.6). In the special case of time lag $\tau=0$, the Eq. (2.7) has the form

$$
\lambda^{2}+\left(\frac{r}{K} x_{0}-\frac{m x_{0} y_{0}}{\left(a+x_{0}\right)^{2}}+R x_{0}\right) \lambda+\frac{a c m^{2} x_{0} y_{0}}{\left(a+x_{0}\right)^{3}}=0,
$$

and using the Routh-Hurwitz theorem [25], we obtain the following result.

Lemma 2.2. Let the system (1.5) have the zero time lag $\tau$. Then

(i) If $r / K+R>m y_{0} /\left(a+x_{0}\right)^{2}$, the positive equilibrium $X_{0}$ is locally asymptotically stable.

(ii) If $r / K+R=m y_{0} /\left(a+x_{0}\right)^{2}$, the positive equilibrium $X_{0}$ is a center.

(iii) If $r / K+R<m y_{0} /\left(a+x_{0}\right)^{2}$, the positive equilibrium $X_{0}$ is unstable.

Proof. Considering the Eq. (2.8), we note that for $r / K+R>m y_{0} /\left(a+x_{0}\right)^{2}$, the real parts of eigenvalues $\lambda$ are always negative. Besides, in the case $r / K+R=m y_{0} /\left(a+x_{0}\right)^{2}$, the Eq. (2.8) has complex conjugate imaginary roots $\pm i \sqrt{a c m^{2} x_{0} y_{0} /\left(a+x_{0}\right)^{3}}$, and if $r / K+$ $R<m y_{0} /\left(a+x_{0}\right)^{2}$, at least one root of (2.8) has a positive real part and the stability follows from [25].

Remark 2.1. If in the case (ii), the roots of Eq. (2.8) satisfy the transversality conditions [16], then the Hopf bifurcation occurs in system (1.6) for the zero time lag $\tau$. The properties of the bifurcation can be studied by the Guckenheimer-Holmes normal form method [16].

If $\tau>0$, we assume that $\pm i \omega, \omega>0$ are the purely imaginary roots of the Eq. (2.7), so that equation

$$
-\omega^{2}+\left[\frac{r}{K} x_{0}(\cos \omega \tau-i \sin \omega \tau)+R x_{0}-\frac{m x_{0} y_{0}}{\left(a+x_{0}\right)^{2}}\right] i \omega+\frac{a c m^{2} x_{0} y_{0}}{\left(a+x_{0}\right)^{3}}=0
$$

holds. Separating real and imaginary parts in this equation, we write

$$
\begin{aligned}
& \sin \omega \tau=\frac{K \omega}{r x_{0}}-\frac{a c m^{2} K y_{0}}{r \omega\left(a+x_{0}\right)^{3}}, \\
& \cos \omega \tau=\frac{m K y_{0}}{r\left(a+x_{0}\right)^{2}}-\frac{K R}{r} .
\end{aligned}
$$

Squaring and summing the Eqs. (2.9) and (2.10) yields

$$
\omega^{4}+\left[\left(\frac{m x_{0} y_{0}}{\left(a+x_{0}\right)^{2}}-R x_{0}\right)^{2}-\frac{r^{2}}{K^{2}} x_{0}^{2}-\frac{2 a c m^{2} x_{0} y_{0}}{\left(a+x_{0}\right)^{3}}\right] \omega^{2}+\frac{a^{2} c^{2} m^{4} x_{0}^{2} y_{0}^{2}}{\left(a+x_{0}\right)^{6}}=0 .
$$

Lemma 2.3. Assuming that the system (1.5) has a positive time lag $\tau$, we have:

(i) If

$$
\frac{r}{K}+R>\frac{m y_{0}}{\left(a+x_{0}\right)^{2}}
$$


and

$$
x_{0}\left(\frac{m y_{0}}{\left(a+x_{0}\right)^{2}}-R\right)^{2}>\frac{r^{2}}{K^{2}} x_{0}+\frac{2 a c m^{2} y_{0}}{\left(a+x_{0}\right)^{3}},
$$

then the roots of (2.7) have negative real parts.

(ii) If

$$
\left[x_{0}\left(\frac{m y_{0}}{\left(a+x_{0}\right)^{2}}-R\right)^{2}-\frac{r^{2}}{K^{2}} x_{0}-\frac{2 a c m^{2} y_{0}}{\left(a+x_{0}\right)^{3}}\right]^{2}>\frac{4 a^{2} c^{2} m^{4} y_{0}^{2}}{\left(a+x_{0}\right)^{6}}
$$

and

$$
x_{0}\left(\frac{m y_{0}}{\left(a+x_{0}\right)^{2}}-R\right)^{2}<\frac{r^{2}}{K^{2}} x_{0}+\frac{2 a c m^{2} y_{0}}{\left(a+x_{0}\right)^{3}},
$$

then (2.11) has two positive roots $\omega^{ \pm}$and the Eq. (2.10) provides the time lags $\tau_{k}^{ \pm}$for the corresponding root - viz.

$$
\tau_{k}^{ \pm}=\frac{1}{\omega^{ \pm}} \arccos \left(\frac{m K y_{0}}{r\left(a+x_{0}\right)^{2}}-\frac{K R}{r}\right)+\frac{2 k \pi}{\omega^{ \pm}}, \quad k=0,1,2, \cdots .
$$

Proof. If

$$
x_{0}\left(\frac{m y_{0}}{\left(a+x_{0}\right)^{2}}-R\right)^{2}>\frac{r^{2}}{K^{2}} x_{0}+\frac{2 a c m^{2} y_{0}}{\left(a+x_{0}\right)^{3}},
$$

then the Eq. (2.11) does not have positive roots, so that (2.7) does not have purely imaginary roots. Assuming that

$$
\frac{r}{K}+R>\frac{m y_{0}}{\left(a+x_{0}\right)^{2}}
$$

we conclude that the both roots of Eq. (2.8) have negative real parts. By Rouche's theorem [35], the real parts of the roots of (2.7) also negative.

In the case (ii), we note that

$$
\Delta=\left[\left(\frac{m x_{0} y_{0}}{\left(a+x_{0}\right)^{2}}-R x_{0}\right)^{2}-\frac{r^{2}}{K^{2}} x_{0}^{2}-\frac{2 a c m^{2} x_{0} y_{0}}{\left(a+x_{0}\right)^{3}}\right]^{2}-\frac{4 a^{2} c^{2} m^{4} x_{0}^{2} y_{0}^{2}}{\left(a+x_{0}\right)^{6}}>0 .
$$

Therefore,

$$
\left(\frac{m x_{0} y_{0}}{\left(a+x_{0}\right)^{2}}-R x_{0}\right)^{2}<\frac{r^{2}}{K^{2}} x_{0}^{2}+\frac{2 a c m^{2} x_{0} y_{0}}{\left(a+x_{0}\right)^{3}},
$$

and the Eq. (2.11) has two positive roots - viz.

$$
\omega^{ \pm}=\left\{\frac{1}{2}\left[\frac{r^{2}}{K^{2}} x_{0}^{2}+\frac{2 a c m^{2} x_{0} y_{0}}{\left(a+x_{0}\right)^{3}}-\left(\frac{m x_{0} y_{0}}{\left(a+x_{0}\right)^{2}}-R x_{0}\right)^{2} \pm \sqrt{\Delta}\right]\right\}^{1 / 2} .
$$

Substituting $\omega^{ \pm}$into Eq. (2.10) and solving the corresponding equation with respect to the time lag $\tau$, we complete the proof. 
Bifurcation in a Differential-Algebra Predator-Prey System with Time Lag Effects

Let us now differentiate the Eq. (2.7) in $\tau$. Then

$$
2 \lambda \frac{\mathrm{d} \lambda}{\mathrm{d} \tau}+\left(R x_{0}-\frac{m x_{0} y_{0}}{\left(a+x_{0}\right)^{2}}\right) \frac{\mathrm{d} \lambda}{\mathrm{d} \tau}+\frac{r}{K} x_{0} e^{-\lambda \tau} \frac{\mathrm{d} \lambda}{\mathrm{d} \tau}+\frac{r}{K} x_{0} \lambda e^{-\lambda \tau}\left(-\lambda-\tau \frac{\mathrm{d} \lambda}{\mathrm{d} \tau}\right)=0 .
$$

It follows that

$$
\left(\frac{\mathrm{d} \lambda}{\mathrm{d} \tau}\right)^{-1}=\frac{2 \lambda e^{\lambda \tau}+\left(R x_{0}-\frac{m x_{0} y_{0}}{\left(a+x_{0}\right)^{2}}\right) e^{\lambda \tau}+\frac{r}{K} x_{0}-\frac{r}{K} x_{0} \lambda \tau}{\frac{r}{K} \lambda^{2} x_{0}}
$$

and, consequently,

$$
\begin{aligned}
\left.\left(\frac{\mathrm{d} \lambda}{\mathrm{d} \tau}\right)^{-1}\right|_{\lambda=i \omega}= & \left(2 i \omega(\cos \omega \tau+i \sin \omega \tau)+\left(R x_{0}-\frac{m x_{0} y_{0}}{\left(a+x_{0}\right)^{2}}\right)(\cos \omega \tau+i \sin \omega \tau)\right. \\
& \left.+\frac{r}{K} x_{0}-\frac{r}{K} x_{0} \tau \omega i\right) /\left(-\frac{r}{K} \omega^{2} x_{0}\right) \\
= & \frac{2 K}{r \omega x_{0}} \sin \omega \tau+\left(\frac{m K y_{0}}{r \omega^{2}\left(a+x_{0}\right)^{2}}-\frac{K R}{r \omega^{2}}\right) \cos \omega \tau-\frac{1}{\omega^{2}} \\
& +i\left[\frac{\tau}{\omega}+\left(\frac{m K y_{0}}{r \omega^{2}\left(a+x_{0}\right)^{2}}-\frac{K R}{r \omega^{2}}\right) \sin \omega \tau-\frac{2 K}{r \omega x_{0}} \cos \omega \tau\right] .
\end{aligned}
$$

Recalling the Eqs. (2.9), (2.10), we rewrite the last expression as

$$
\begin{aligned}
\left.\operatorname{Re}\left(\frac{\mathrm{d} \lambda}{\mathrm{d} \tau}\right)^{-1}\right|_{\lambda=i \omega}= & \frac{2 K}{r \omega x_{0}}\left(\frac{K \omega}{r x_{0}}-\frac{a c m^{2} K y_{0}}{r \omega\left(a+x_{0}\right)^{3}}\right) \\
& +\left(\frac{m K y_{0}}{r \omega^{2}\left(a+x_{0}\right)^{2}}-\frac{K R}{r \omega^{2}}\right)\left(\frac{m K y_{0}}{r\left(a+x_{0}\right)^{2}}-\frac{K R}{r}\right)-\frac{1}{\omega^{2}} \\
= & \frac{K^{2}}{r^{2} \omega^{2} x_{0}^{2}}\left[2 \omega^{2}-\frac{2 a c m^{2} x_{0} y_{0}}{\left(a+x_{0}\right)^{3}}+\left(\frac{m x_{0} y_{0}}{\left(a+x_{0}\right)^{2}}-R x_{0}\right)^{2}-\frac{r^{2} x_{0}^{2}}{K^{2}}\right] .
\end{aligned}
$$

It follows that

$$
\begin{aligned}
& \operatorname{sign}\left\{\operatorname{Re}\left(\frac{\mathrm{d} \lambda}{\mathrm{d} \tau}\right)\right\}_{\lambda=i \omega}=\operatorname{sign}\left\{\operatorname{Re}\left(\frac{\mathrm{d} \lambda}{\mathrm{d} \tau}\right)^{-1}\right\}_{\lambda=i \omega} \\
= & \operatorname{sign}\left\{2 \omega^{2}-\frac{2 a c m^{2} x_{0} y_{0}}{\left(a+x_{0}\right)^{3}}+\left(\frac{m x_{0} y_{0}}{\left(a+x_{0}\right)^{2}}-R x_{0}\right)^{2}-\frac{r^{2} x_{0}^{2}}{K^{2}}\right\},
\end{aligned}
$$

which yields the transversality conditions

$$
\operatorname{sign}\left\{\operatorname{Re}\left(\frac{\mathrm{d} \lambda}{\mathrm{d} \tau}\right)\right\}_{\tau=\tau_{k}^{+}, \omega=\omega^{+}}>0 \text { and } \operatorname{sign}\left\{\operatorname{Re}\left(\frac{\mathrm{d} \lambda}{\mathrm{d} \tau}\right)\right\}_{\tau=\tau_{k}^{-}, \omega=\omega^{-}}<0 .
$$

Taking into account Refs. $[10,17]$ and using the previous lemma, we obtain the following result. 
Theorem 2.1. Assuming that the system (1.5) has a positive time lag $\tau$ and

$$
\frac{r}{K}+R>\frac{m y_{0}}{\left(a+x_{0}\right)^{2}}
$$

we have:

(i) If

$$
x_{0}\left(\frac{m y_{0}}{\left(a+x_{0}\right)^{2}}-R\right)^{2}>\frac{r^{2}}{K^{2}} x_{0}+\frac{2 a c m^{2} y_{0}}{\left(a+x_{0}\right)^{3}},
$$

then the positive equilibrium $X_{0}$ is locally asymptotically stable.

(ii) If

$$
\left[x_{0}\left(\frac{m y_{0}}{\left(a+x_{0}\right)^{2}}-R\right)^{2}-\frac{r^{2}}{K^{2}} x_{0}-\frac{2 a c m^{2} y_{0}}{\left(a+x_{0}\right)^{3}}\right]^{2}>\frac{4 a^{2} c^{2} m^{4} y_{0}^{2}}{\left(a+x_{0}\right)^{6}}
$$

and

$$
x_{0}\left(\frac{m y_{0}}{\left(a+x_{0}\right)^{2}}-R\right)^{2}<\frac{r^{2}}{K^{2}} x_{0}+\frac{2 a c m^{2} y_{0}}{\left(a+x_{0}\right)^{3}},
$$

then there exists a positive integer $N$, such that the positive equilibrium $X_{0}$ is locally asymptotically stable for

$$
\tau \in\left[0, \tau_{0}^{+}\right) \bigcup\left(\tau_{0}^{-}, \tau_{1}^{+}\right) \bigcup\left(\tau_{1}^{-}, \tau_{2}^{+}\right) \bigcup\left(\tau_{2}^{-}, \tau_{3}^{+}\right) \bigcup \cdots \bigcup\left(\tau_{N-1}^{-}, \tau_{N}^{+}\right)
$$

and unstable for

$$
\tau \in\left(\tau_{0}^{+}, \tau_{0}^{-}\right) \bigcup\left(\tau_{1}^{+}, \tau_{1}^{-}\right) \bigcup\left(\tau_{2}^{+}, \tau_{2}^{-}\right) \bigcup \cdots \bigcup\left(\tau_{N-1}^{+}, \tau_{N-1}^{-}\right) \bigcup\left(\tau_{N}^{+},+\infty\right) .
$$

The proof of Theorem 2.1 is similar to the corresponding proofs in $[10,17]$ and is omitted here.

\section{Properties of Hopf Bifurcations}

In this section, we use the center manifold theorem [18] to study the direction, stability and period of Hopf bifurcations. In order to determine the leading order terms in the theorem mentioned, we first consider the second-order Taylor expansions of the parameterised system (2.5) - i.e.

$$
\begin{aligned}
\dot{y}_{1}= & f_{1 y_{1}}\left(\bar{X}_{0}\right) y_{1}+f_{1 y_{2}}\left(\bar{X}_{0}\right) y_{2}+\frac{1}{2} f_{1 y_{1} y_{1}}\left(\bar{X}_{0}\right) y_{1}^{2}+f_{1 y_{1} y_{2}}\left(\bar{X}_{0}\right) y_{1} y_{2} \\
& +\frac{1}{2} f_{1 y_{2} y_{2}}\left(\bar{X}_{0}\right) y_{2}^{2}+o\left(|Y|^{3}\right), \\
\dot{y}_{2}= & f_{2 y_{1}}\left(\bar{X}_{0}\right) y_{1}+f_{2 y_{2}}\left(\bar{X}_{0}\right) y_{2}+\frac{1}{2} f_{2 y_{1} y_{1}}\left(\bar{X}_{0}\right) y_{1}^{2}+f_{2 y_{1} y_{2}}\left(\bar{X}_{0}\right) y_{1} y_{2} \\
& +\frac{1}{2} f_{2 y_{2} y_{2}}\left(\bar{X}_{0}\right) y_{2}^{2}+o\left(|Y|^{3}\right) .
\end{aligned}
$$


To compute the coefficients in the expansions (3.1), we use the Eqs. (2.4) so that

$$
\begin{aligned}
D_{\bar{X}} f_{1}(\bar{X}) & =\left(r-\frac{r}{K} x(t-\tau)-\frac{m y}{a+x}-(R x+\bar{E})-\frac{r}{K} e^{-\lambda \tau} x+\frac{m x y}{(a+x)^{2}}-R x,-\frac{m x}{a+x},-x\right), \\
D_{\bar{X}} f_{2}(\bar{X}) & =\left(\frac{a c m y}{(a+x)^{2}},-d+\frac{c m x}{a+x}, 0\right), \\
D_{\bar{X}} g(\bar{X}) & =\left(\frac{b c(\bar{E}+2 R x)-e R[c+(\bar{E}+R x) x]^{2}}{[c+(\bar{E}+R x) x]^{2}}, 0, \frac{b c x-e[c+(\bar{E}+R x) x]^{2}}{[c+(\bar{E}+R x) x]^{2}}\right) .
\end{aligned}
$$

Besides, the Eqs. A.5 and A.6 in the Appendix imply

$$
\begin{aligned}
& D_{Y} \psi(Y)=\left(D_{y_{1}} \psi(Y), D_{y_{2}} \psi(Y)\right) \\
= & \left(\begin{array}{c}
D_{\bar{X}} g(\bar{X}) \\
U_{0}^{T}
\end{array}\right)^{-1}\left(\begin{array}{c}
0 \\
I_{2}
\end{array}\right)=\left(\begin{array}{cc}
1 & 0 \\
0 & 1 \\
\frac{b c(\bar{E}+2 R x)-e R[c+(\bar{E}+R x) x]^{2}}{e[c+(\bar{E}+R x) x]^{2}-b c x} & 0
\end{array}\right),
\end{aligned}
$$

and the Eqs. (2.5), (3.2) and (3.3) yield

$$
\begin{aligned}
f_{1 y_{1}}(\bar{X})= & D_{\bar{X}} f_{1}(\bar{X}) D_{y_{1}} \psi(Y)=r-\frac{r}{K} x(t-\tau)-\frac{m y}{a+x} \\
& -(R x+\bar{E})-\frac{r}{K} x e^{-\lambda \tau}+\frac{m x y}{(a+x)^{2}}-R x \\
& -\frac{b c x(\bar{E}+2 R x)-e R x[c+(\bar{E}+R x) x]^{2}}{e[c+(\bar{E}+R x) x]^{2}-b c x} \\
f_{1 y_{2}}(\bar{X})= & D_{\bar{X}} f_{1}(\bar{X}) D_{y_{2}} \psi(Y)=-\frac{m x}{a+x} \\
f_{2 y_{1}}(\bar{X})= & D_{\bar{X}} f_{2}(\bar{X}) D_{y_{1}} \psi(Y)=\frac{a c m y}{(a+x)^{2}} \\
f_{2 y_{2}}(\bar{X})= & D_{\bar{X}} f_{2}(\bar{X}) D_{y_{2}} \psi(Y)=-d+\frac{c m x}{a+x} .
\end{aligned}
$$

Substituting $\bar{X}_{0}$ into (3.4) leads to the representations

$$
\begin{aligned}
f_{1 y_{1}}\left(\bar{X}_{0}\right)= & -\frac{r}{K} e^{-\lambda \tau} x_{0}+\frac{m x_{0} y_{0}}{\left(a+x_{0}\right)^{2}}-R x_{0} \\
& -\frac{b c x_{0}\left(E_{0}+R x_{0}\right)-e R x_{0}\left(c+E_{0} x_{0}\right)^{2}}{e\left(c+E_{0} x_{0}\right)^{2}-b c x_{0}}, \\
f_{1 y_{2}}\left(\bar{X}_{0}\right)= & -\frac{m x_{0}}{a+x_{0}}, \quad f_{2 y_{1}}\left(\bar{X}_{0}\right)=\frac{a c m y_{0}}{\left(a+x_{0}\right)^{2}}, \\
f_{2 y_{2}}\left(\bar{X}_{0}\right)= & 0 .
\end{aligned}
$$

We observe that the equilibrium $\bar{X}_{0}$ of DAE system (2.3) corresponds to the equilibrium $Y=0$ of the locally parameterised system (2.5), and $R=-b c E_{0} /\left[b c x_{0}-e\left(c+E_{0} x_{0}\right)^{2}\right]$. 
Therefore, it follows from (3.3) and (3.4) that

$$
\begin{aligned}
& D_{\bar{X}} f_{1 y_{1}}\left(\bar{X}_{0}\right)=\left(\frac{2 a m y_{0}}{\left(a+x_{0}\right)^{3}}-\frac{2 r}{K} e^{-\lambda \tau}-2 R\right. \\
& +\frac{2 b c e x_{0} E_{0}\left(c+E_{0} x_{0}\right)\left(E_{0}+R x_{0}\right)+2 R b^{2} c^{2} x_{0}^{2}}{\left[e\left(c+E_{0} x_{0}\right)^{2}-b c x_{0}\right]^{2}} \\
& -\frac{e\left(c+E_{0} x_{0}\right)^{2}\left[\left(b c E_{0}+3 b c R x_{0}\right)-e R\left(c+E_{0} x_{0}\right)^{2}\right]}{\left[e\left(c+E_{0} x_{0}\right)^{2}-b c x_{0}\right]^{2}}, \\
& \left.-\frac{a m}{\left(a+x_{0}\right)^{2}},\left.\frac{\partial f_{1 y_{1}}}{\partial \bar{E}}\right|_{\bar{X}=\bar{X}_{0}}\right) \text {, } \\
& D_{\bar{X}} f_{1 y_{2}}\left(\bar{X}_{0}\right)=\left(-\frac{a m}{\left(a+x_{0}\right)^{2}}, 0,0\right) \text {, } \\
& D_{\bar{X}} f_{2 y_{1}}\left(\bar{X}_{0}\right)=\left(-\frac{2 a c m y_{0}}{\left(a+x_{0}\right)^{3}}, \frac{a c m}{\left(a+x_{0}\right)^{2}}, 0\right) \text {, } \\
& D_{\bar{X}} f_{2 y_{2}}\left(\bar{X}_{0}\right)=\left(\frac{a c m}{\left(a+x_{0}\right)^{2}}, 0,0\right) \text {, } \\
& D_{Y} \psi(0)=\left(D_{y_{1}} \psi(0), D_{y_{2}} \psi(0)\right) \\
& =\left(\begin{array}{cc}
1 & 0 \\
0 & 1 \\
\frac{b c\left(E_{0}+R x_{0}\right)-e R\left(c+E_{0} x_{0}\right)^{2}}{e\left(c+E_{0} x_{0}\right)^{2}-b c x_{0}} & 0
\end{array}\right)=\left(\begin{array}{ll}
1 & 0 \\
0 & 1 \\
0 & 0
\end{array}\right) .
\end{aligned}
$$

Using the Eqs. (2.5) and (3.6), we write

$$
\begin{aligned}
f_{1 y_{1} y_{1}}\left(\bar{X}_{0}\right)= & D_{\bar{X}} f_{1 y_{1}}\left(\bar{X}_{0}\right) D_{y_{1}} \psi(0)=\frac{2 a m y_{0}}{\left(a+x_{0}\right)^{3}}-\frac{2 r}{K} e^{-\lambda \tau}-2 R \\
& +\frac{2 b c e x_{0} E_{0}\left(c+E_{0} x_{0}\right)\left(E_{0}+R x_{0}\right)+2 R b^{2} c^{2} x_{0}^{2}}{\left[e\left(c+E_{0} x_{0}\right)^{2}-b c x_{0}\right]^{2}} \\
& -\frac{e\left(c+E_{0} x_{0}\right)^{2}\left[\left(b c E_{0}+3 b c R x_{0}\right)-e R\left(c+E_{0} x_{0}\right)^{2}\right]}{\left[e\left(c+E_{0} x_{0}\right)^{2}-b c x_{0}\right]^{2}} \\
f_{1 y_{1} y_{2}}\left(\bar{X}_{0}\right)= & D_{\bar{X}} f_{1 y_{1}}\left(\bar{X}_{0}\right) D_{y_{2}} \psi(0)=-\frac{a m}{\left(a+x_{0}\right)^{2}} \\
f_{1 y_{2} y_{2}}\left(\bar{X}_{0}\right)= & D_{\bar{X}} f_{1 y_{2}}\left(\bar{X}_{0}\right) D_{y_{2}} \psi(0)=0 \\
f_{2 y_{1} y_{1}}\left(\bar{X}_{0}\right)= & D_{\bar{X}} f_{2 y_{1}}\left(\bar{X}_{0}\right) D_{y_{1}} \psi(0)=-\frac{2 a c m y_{0}}{\left(a+x_{0}\right)^{3}} \\
f_{2 y_{1} y_{2}}\left(\bar{X}_{0}\right)= & D_{\bar{X}} f_{2 y_{1}}\left(\bar{X}_{0}\right) D_{y_{2}} \psi(0)=\frac{a c m}{\left(a+x_{0}\right)^{2}} \\
f_{2 y_{2} y_{2}}\left(\bar{X}_{0}\right)= & D_{\bar{X}} f_{2 y_{2}}\left(\bar{X}_{0}\right) D_{y_{2}} \psi(0)=0
\end{aligned}
$$

If we now substitute the coefficients of (3.5), (3.7) into the expansions (3.1), it takes the form 


$$
\begin{aligned}
\dot{y}_{1}(t)= & \left(\frac{m x_{0} y_{0}}{\left(a+x_{0}\right)^{2}}-R x_{0}-\frac{b c x_{0}\left(E_{0}+R x_{0}\right)-e R x_{0}\left(c+E_{0} x_{0}\right)^{2}}{e\left(c+E_{0} x_{0}\right)^{2}-b c x_{0}}\right) y_{1}(t) \\
& -\frac{r}{K} x_{0} y_{1}(t-\tau)-\frac{m x_{0}}{a+x_{0}} y_{2}(t)-\frac{a m}{\left(a+x_{0}\right)^{2}} y_{1}(t) y_{2}(t) \\
& +\left(\frac{a m y_{0}}{\left(a+x_{0}\right)^{3}}-R+\frac{b c e x_{0} E_{0}\left(c+E_{0} x_{0}\right)\left(E_{0}+R x_{0}\right)+R b^{2} c^{2} x_{0}^{2}}{\left[e\left(c+E_{0} x_{0}\right)^{2}-b c x_{0}\right]^{2}}\right) y_{1}^{2}(t) \\
& -\frac{e\left(c+E_{0} x_{0}\right)^{2}\left[\left(b c E_{0}+3 b c R x_{0}\right)-e R\left(c+E_{0} x_{0}\right)^{2}\right]}{2\left[e\left(c+E_{0} x_{0}\right)^{2}-b c x_{0}\right]^{2}} \\
& -\frac{r}{K} y_{1}^{2}(t-\tau)+o\left(|Y|^{3}\right), \\
\dot{y}_{2}(t)= & \frac{a c m y_{0}}{\left(a+x_{0}\right)^{2}} y_{1}(t)+\frac{a c m}{\left(a+x_{0}\right)^{2}} y_{1}(t) y_{2}(t)-\frac{a c m y_{0}}{\left(a+x_{0}\right)^{3}} y_{1}^{2}(t)+o\left(|Y|^{3}\right) .
\end{aligned}
$$

Theorem 2.1 shows that if the time lag $\tau$ takes bifurcation values $\tau_{n}^{ \pm}, n=0,1,2, \cdots, N$, Hopf bifurcations can occur at positive equilibrium $X_{0}$. From now on we always assume that $\tau_{n}$ is a bifurcation value and there is a Hopf bifurcation at $\tau_{n}$. Moreover, let $i \omega$ be the purely imaginary root of the Eq. (2.7) corresponding to $\tau_{n}$. Introducing new parameter $\mu:=\tau-\tau_{n}$, we note that $\mu=0$ is the Hopf bifurcation value of the differential-algebra predator-prey system (1.5) at its positive equilibrium $X_{0}$, and setting $y_{1}(t):=x(\tau t)-$ $x_{0}, y_{2}(t):=y(\tau t)-y_{0}$, we write the Eqs. (3.8) as the system of retarded functional differential equations in the phase space $C\left([-1,0], \mathbb{R}^{2}\right)$ - i.e.

$$
\dot{Y}(t)=L_{\mu}\left(Y_{t}\right)+F\left(\mu, Y_{t}\right)
$$

where

$$
Y(t)=\left(y_{1}(t), y_{2}(t)\right)^{T}, \quad Y_{t}=Y(t+\theta)=\left(y_{1}(t+\theta), y_{2}(t+\theta)\right), \quad \theta \in[-1,0] .
$$

For $\Phi(\theta)=\left(\Phi_{1}(\theta), \Phi_{2}(\theta)\right) \in C\left([-1,0], \mathbb{R}^{2}\right)$, we define

$$
\begin{aligned}
L_{\mu} \Phi= & \left(\tau_{n}+\mu\right)\left(\begin{array}{cc}
\frac{m x_{0} y_{0}}{\left(a+x_{0}\right)^{2}}-R x_{0}-\frac{b c x_{0}\left(E_{0}+R x_{0}\right)-e R x_{0}\left(c+E_{0} x_{0}\right)^{2}}{e\left(c+E_{0} x_{0}\right)^{2}-b c x_{0}} & -\frac{m x_{0}}{a+x_{0}} \\
\frac{a c m y_{0}}{\left(a+x_{0}\right)^{2}} & 0
\end{array}\right) \Phi^{T}(0) \\
& +\left(\tau_{n}+\mu\right)\left(\begin{array}{cc}
-\frac{r}{K} x_{0} & 0 \\
0 & 0
\end{array}\right) \Phi^{T}(-1),
\end{aligned}
$$

and

$$
F(\mu, \Phi(\theta))=\left(\tau_{n}+\mu\right)\left(\begin{array}{l}
F_{11} \\
F_{22}
\end{array}\right)
$$

where

$$
F_{11}=-\frac{a m}{\left(a+x_{0}\right)^{2}} \Phi_{1}(0) \Phi_{2}(0)+\left(\frac{a m y_{0}}{\left(a+x_{0}\right)^{3}}-R+\frac{b c e x_{0} E_{0}\left(c+E_{0} x_{0}\right)\left(E_{0}+R x_{0}\right)+R b^{2} c^{2} x_{0}^{2}}{\left[e\left(c+E_{0} x_{0}\right)^{2}-b c x_{0}\right]^{2}}\right.
$$




$$
\begin{aligned}
& \left.-\frac{e\left(c+E_{0} x_{0}\right)^{2}\left[\left(b c E_{0}+3 b c R x_{0}\right)-e R\left(c+E_{0} x_{0}\right)^{2}\right]}{2\left[e\left(c+E_{0} x_{0}\right)^{2}-b c x_{0}\right]^{2}}\right) \Phi_{1}^{2}(0)-\frac{r}{K} \Phi_{1}^{2}(-1)+\cdots, \\
F_{22}= & \frac{a c m}{\left(a+x_{0}\right)^{2}} \Phi_{1}(0) \Phi_{2}(0)-\frac{a c m y_{0}}{\left(a+x_{0}\right)^{3}} \Phi_{1}^{2}(0)+\cdots .
\end{aligned}
$$

It is clear that $L_{\mu}$ is a continuous linear operator acting from the space $C\left([-1,0], \mathbb{R}^{2}\right)$ into $\mathbb{R}^{2}$. According to the Riesz representation theorem [41], one has

$$
L_{\mu} \Phi=\int_{-1}^{0}[\mathrm{~d} \eta(\theta, \mu)] \Phi(\theta) \text { for } \Phi(\theta) \in C\left([-1,0], \mathbb{R}^{2}\right),
$$

where $\eta(\theta, \mu)$ is a $2 \times 2$ matrix-function of bounded variation on the interval $\theta \in[-1,0]$. More precisely,

$$
\begin{aligned}
\eta(\theta, \mu)= & \left(\tau_{n}+\mu\right)\left(\begin{array}{cc}
\frac{m x_{0} y_{0}}{\left(a+x_{0}\right)^{2}}-R x_{0}-\frac{b c x_{0}\left(E_{0}+R x_{0}\right)-e R x_{0}\left(c+E_{0} x_{0}\right)^{2}}{e\left(c+E_{0} x_{0}\right)^{2}-b c x_{0}} & -\frac{m x_{0}}{a+x_{0}} \\
\frac{a c m y_{0}}{\left(a+x_{0}\right)^{2}} & 0
\end{array}\right) \\
& \times \delta(\theta)+\left(\tau_{n}+\mu\right)\left(\begin{array}{cc}
\frac{r}{K} x_{0} & 0 \\
0 & 0
\end{array}\right) \delta(\theta+1),
\end{aligned}
$$

and

$$
\delta(\theta):= \begin{cases}0, & \theta \in[-1,0) \\ 1, & \theta=0\end{cases}
$$

is the delta function.

On the space $C^{1}\left([-1,0], \mathbb{R}^{2}\right)$ we define operators $A(\mu)$ and $R(\mu)$ by

$$
\begin{aligned}
& A(\mu) \Phi(\theta):=\left\{\begin{array}{l}
\frac{\mathrm{d} \Phi(\theta)}{\mathrm{d} \theta}, \quad-1 \leq \theta<0, \\
\int_{-1}^{0} \mathrm{~d} \eta(\theta, \mu) \Phi(\theta), \quad \theta=0,
\end{array}\right. \\
& R(\mu) \Phi(\theta):=\left\{\begin{array}{l}
0,-1 \leq \theta<0, \\
F(\mu, \Phi(\theta)), \quad \theta=0 .
\end{array}\right.
\end{aligned}
$$

Then the system (3.9) can be written as

$$
\dot{Y}(t)=A(\mu) Y_{t}+R(\mu) Y_{t} .
$$

According to the considerations in Section 2, the operator $A(0)$ has a pair of simple purely imaginary eigenvalues $\pm i \omega \tau_{n}$.

Consider the bilinear form

$$
<\Psi(s), \Phi(\theta)>=\bar{\Psi}(0) \Phi(0)-\int_{\theta=-1}^{0} \int_{\xi=0}^{\theta} \bar{\Psi}(\xi-\theta) \mathrm{d} \eta(\theta) \Phi(\xi) \mathrm{d} \xi
$$


where $\Psi(s) \in C^{1}\left([0,1],\left(\mathbb{R}^{2}\right)^{*}\right), \Phi(\theta) \in C^{1}\left([-1,0], \mathbb{R}^{2}\right), \eta(\theta)=\eta(\theta, 0)$, and on the space $C^{1}\left([0,1],\left(\mathbb{R}^{2}\right)^{*}\right.$, we define a formal adjoint operator $A^{*}$ for the operator $A(0)$ by

$$
A^{*} \Psi(s)=\left\{\begin{array}{l}
-\frac{\mathrm{d} \Psi(s)}{d s}, \quad 0<s \leq 1, \\
\int_{-1}^{0} \mathrm{~d} \eta^{T}(s, 0) \Psi(-s), \quad s=0 .
\end{array}\right.
$$

Consequently, $A(0)$ and $A^{*}$ are reciprocally adjoint [18]. Therefore, the purely imaginary roots $\pm i \omega \tau_{n}$ of the operator $A(0)$ are simultaneously the eigenvalues of the operator $A^{*}$.

We now determine the corresponding eigenvectors of the operators $A(0)$ and $A^{*}$. Let $q(\theta)=\left(1, q_{2}\right)^{T} e^{i \omega \tau_{n} \theta}, \theta \in[-1,0]$ and $q^{*}(s)=(1 / D)\left(q_{2}^{*}, 1\right) e^{i \omega \tau_{n} s}, s \in[0,1]$ be, respectively, the eigenvectors of the operators $A(0)$ and $A^{*}$, corresponding to the eigenvalues $i \omega \tau_{n}$ and $-i \omega \tau_{n}$, so that $A(0) q(\theta)=i \omega \tau_{n} q(\theta)$ and $A^{*} q^{*}(s)=-i \omega \tau_{n} q^{*}(s)$. Straightforward calculations show

$$
\begin{aligned}
q_{2}= & -\frac{\left(a+x_{0}\right) i \omega}{m x_{0}}-\frac{R\left(a+x_{0}\right)}{m} \\
& -\frac{\left(a+x_{0}\right)\left[b c\left(E_{0}+R x_{0}\right)-e R\left(c+E_{0} x_{0}\right)^{2}\right]}{m\left[e\left(c+E_{0} x_{0}\right)^{2}-b c x_{0}\right]} \\
& +\frac{y_{0}}{a+x_{0}}-\frac{r\left(a+x_{0}\right)}{m K} e^{-i \omega \tau_{n}}, \\
q_{2}^{*}= & \frac{i \omega\left(a+x_{0}\right)}{m x_{0}} .
\end{aligned}
$$

We also observe that

$$
\begin{aligned}
<q^{*}(s), q(\theta)> & =\overline{q^{*}}(0) q(0)-\int_{\theta=-1}^{0} \int_{\xi=0}^{\theta} \overline{q^{*}}(\xi-\theta) \mathrm{d} \eta(\theta) q(\xi) \mathrm{d} \xi \\
& =\frac{1}{\bar{D}}\left\{q_{2}+\bar{q}_{2}^{*}-\int_{\theta=-1}^{0}\left(\overline{q_{2}^{*}}, 1\right) e^{i \omega \tau_{n} \theta} \theta \mathrm{d} \eta(\theta)\left(1, q_{2}\right)^{T}\right\} \\
& =\frac{1}{\bar{D}}\left(q_{2}+\bar{q}_{2}^{*}+\frac{r \tau_{n} \bar{q}_{2}^{*} x_{0} e^{-i \omega \tau_{n}}}{K}\right) .
\end{aligned}
$$

Therefore, to satisfy the requirement $\left\langle q^{*}(s), q(\theta)\right\rangle=1$, one has to set $\bar{D}=q_{2}+\bar{q}_{2}^{*}+$ $\left(r \tau_{n} \bar{q}_{2}^{*} x_{0} e^{-i \omega \tau_{n}} / K\right)$. Analogous considerations show that $<q^{*}(s), \bar{q}(\theta)>=0$.

Now we want to find the coordinates of the center manifold $C_{0}$ at the Hopf bifurcation value $\mu=0-$ i.e. for $\tau=\tau_{n}$. Our considerations are based on the ideas from [18]. Setting

$$
z(t):=<q^{*}, Y_{t}>, \quad W(t, \theta):=Y_{t}-2 \operatorname{Re}\{z(t) q(\theta)\}
$$

on the center manifold $C_{0}$, we have

$$
W(t, \theta)=W(z(t), \bar{z}(t), \theta)=W_{20}(\theta) \frac{z^{2}}{2}+W_{11}(\theta) z \bar{z}+W_{02}(\theta) \frac{\bar{z}^{2}}{2}+W_{30}(\theta) \frac{z^{3}}{6}+\cdots,
$$


where $z$ and $\bar{z}$ are the local coordinates for the manifold $C_{0}$ in the directions $q$ and $\bar{q}^{*}$. Taking into account the Eqs. (3.11) and (3.12), we observe that $W(z(t), \bar{z}(t), \theta)$ is a realvalued function if so is the function $Y_{t}$. If $\mu=0$ and $Y_{t} \in C_{0}$ is a real-valued solution, then it follows from the Eqs. (3.10)-(3.12) that

$$
\dot{z}(t)=i \omega \tau_{n} z(t)+\bar{q}^{*}(0) F_{0}(z, \bar{z}):=i \omega \tau_{n} z(t)+g(z, \bar{z}),
$$

where

$$
g(z, \bar{z})=g_{20}(\theta) \frac{z^{2}}{2}+g_{11}(\theta) z \bar{z}+g_{02}(\theta) \frac{\bar{z}^{2}}{2}+g_{21}(\theta) \frac{z^{2} \bar{z}}{2}+\cdots
$$

Further, the Eq. (3.13) yields

$$
g(z, \bar{z})=\bar{q}^{*}(0) F_{0}(z, \bar{z})=\frac{\tau_{n}}{\bar{D}}\left(1, \bar{q}_{2}^{*}\right)\left(\begin{array}{l}
F_{11}^{0} \\
F_{22}^{0}
\end{array}\right)=\frac{\tau_{n}}{\bar{D}}\left(F_{11}^{0}+\bar{q}_{2}^{*} F_{22}^{0}\right),
$$

where

$$
\begin{aligned}
F_{11}^{0}= & -\frac{a m}{\left(a+x_{0}\right)^{2}} y_{1 t}(0) y_{2 t}(0) \\
& +\left(\frac{a m y_{0}}{\left(a+x_{0}\right)^{3}}-R+\frac{b c e x_{0} E_{0}\left(c+E_{0} x_{0}\right)\left(E_{0}+R x_{0}\right)+R b^{2} c^{2} x_{0}^{2}}{\left[e\left(c+E_{0} x_{0}\right)^{2}-b c x_{0}\right]^{2}}\right. \\
& \left.-\frac{e\left(c+E_{0} x_{0}\right)^{2}\left[\left(b c E_{0}+3 b c R x_{0}\right)-e R\left(c+E_{0} x_{0}\right)^{2}\right]}{2\left[e\left(c+E_{0} x_{0}\right)^{2}-b c x_{0}\right]^{2}}\right) y_{1 t}^{2}(0) \\
& -\frac{r}{K} y_{1 t}^{2}(-1)+\cdots, \\
F_{22}^{0}= & \frac{a c m}{\left(a+x_{0}\right)^{2}} y_{1 t}(0) y_{2 t}(0)-\frac{a c m y_{0}}{\left(a+x_{0}\right)^{3}} y_{1 t}^{2}(0)+\cdots .
\end{aligned}
$$

Combining this with (3.12), we obtain

$$
\begin{aligned}
g(z, \bar{z})= & \frac{\tau_{n}}{\bar{Q}}\left\{\left[\frac{a m q_{2}^{*} y_{0}}{\left(a+x_{0}\right)^{3}}-R \bar{q}_{2}^{*}+\frac{b c e \bar{q}_{2}^{*} x_{0} E_{0}\left(c+E_{0} x_{0}\right)\left(E_{0}+R x_{0}\right)+R b^{2} c^{2} \bar{q}_{2}^{*} x_{0}^{2}}{\left[e\left(c+E_{0} x_{0}\right)^{2}-b c x_{0}\right]^{2}}\right.\right. \\
& \left.-\frac{e q_{2}^{*}\left(c+E_{0} x_{0}\right)^{2}\left[\left(b c E_{0}+3 b c R x_{0}\right)-e R\left(c+E_{0} x_{0}\right)^{2}\right]}{2\left[e\left(c+E_{0} x_{0}\right)^{2}-b c x_{0}\right]^{2}}\right] \\
& \times\left[z+\bar{z}+W_{20}^{(1)}(0) \frac{z^{2}}{2}+W_{11}^{(1)}(0) z \bar{z}+W_{02}^{(1)}(0) \frac{\bar{z}^{2}}{2}\right]^{2} \\
& -\frac{r \bar{q}_{2}^{*}}{K}\left[z e^{-i \omega \tau_{n} \theta}+\bar{z} e^{i \omega \tau_{n} \theta}+W_{20}^{(1)}(-1) \frac{z^{2}}{2}+W_{11}^{(1)}(-1) z \bar{z}+W_{02}^{(1)}(-1) \frac{\bar{z}^{2}}{2}\right]^{2} \\
& -\frac{a m q_{2}^{*}}{\left(a+x_{0}\right)^{2}}\left[z+\bar{z}+W_{20}^{(1)}(0) \frac{z^{2}}{2}+W_{11}^{(1)}(0) z \bar{z}+W_{02}^{(1)}(0) \frac{\bar{z}^{2}}{2}\right] \\
& \times\left[q_{2} z+\bar{q}_{2} \bar{z}+W_{20}^{(2)}(0) \frac{z^{2}}{2}+W_{11}^{(2)}(0) z \bar{z}+W_{02}^{(2)}(0) \frac{\bar{z}^{2}}{2}\right]
\end{aligned}
$$


Bifurcation in a Differential-Algebra Predator-Prey System with Time Lag Effects

$$
\begin{aligned}
& -\frac{a c m y_{0}}{\left(a+x_{0}\right)^{3}}\left[z+\bar{z}+W_{20}^{(1)}(0) \frac{z^{2}}{2}+W_{11}^{(1)}(0) z \bar{z}+W_{02}^{(1)}(0) \frac{\bar{z}^{2}}{2}\right]^{2} \\
& +\frac{a c m}{\left(a+x_{0}\right)^{2}}\left[z+\bar{z}+W_{20}^{(1)}(0) \frac{z^{2}}{2}+W_{11}^{(1)}(0) z \bar{z}+W_{02}^{(1)}(0) \frac{\bar{z}^{2}}{2}\right] \\
& \left.\times\left[q_{2} z+\bar{q}_{2} \bar{z}+W_{20}^{(2)}(0) \frac{z^{2}}{2}+W_{11}^{(2)}(0) z \bar{z}+W_{02}^{(2)}(0) \frac{\bar{z}^{2}}{2}\right]+\cdots\right\},
\end{aligned}
$$

and, consequently,

$$
\begin{aligned}
& g(z, \bar{z})=\frac{\tau_{n}}{\bar{D}}\left\{z ^ { 2 } \left[\frac{a m \bar{q}_{2}^{*} y_{0}}{\left(a+x_{0}\right)^{3}}-\frac{a m q_{2} \bar{q}_{2}^{*}}{\left(a+x_{0}\right)^{2}}-R \bar{q}_{2}^{*}-\frac{r \bar{q}_{2}^{*}}{K} e^{-2 i \omega \tau_{n} \theta}+\frac{a c m q_{2}}{\left(a+x_{0}\right)^{2}}-\frac{a c m y_{0}}{\left(a+x_{0}\right)^{3}}\right.\right. \\
& +\frac{b c e q_{2}^{*} x_{0} E_{0}\left(c+E_{0} x_{0}\right)\left(E_{0}+R x_{0}\right)+b^{2} c^{2} R \bar{q}_{2}^{*} x_{0}^{2}}{\left[e\left(c+E_{0} x_{0}\right)^{2}-b c x_{0}\right]^{2}} \\
& \left.-\frac{e q_{2}^{*}\left(c+E_{0} x_{0}\right)^{2}\left[\left(b c E_{0}+3 b c R x_{0}\right)-e R\left(c+E_{0} x_{0}\right)^{2}\right]}{2\left[e\left(c+E_{0} x_{0}\right)^{2}-b c x_{0}\right]^{2}}\right] \\
& +z \bar{z}\left[\frac{2 a m \bar{q}_{2}^{*} y_{0}}{\left(a+x_{0}\right)^{3}}-\frac{2 a m \bar{q}_{2}^{*} \operatorname{Re}\left(q_{2}\right)}{\left(a+x_{0}\right)^{2}}-2 R \bar{q}_{2}^{*}-\frac{2 r \bar{q}_{2}^{*}}{K}+\frac{2 a c m \operatorname{Re}\left(q_{2}\right)}{\left(a+x_{0}\right)^{2}}-\frac{2 a c m y_{0}}{\left(a+x_{0}\right)^{3}}\right. \\
& +\frac{2 b c e q_{2}^{*} x_{0} E_{0}\left(c+E_{0} x_{0}\right)\left(E_{0}+R x_{0}\right)+2 b^{2} c^{2} R \bar{q}_{2}^{*} x_{0}^{2}}{\left[e\left(c+E_{0} x_{0}\right)^{2}-b c x_{0}\right]^{2}} \\
& \left.-\frac{e q_{2}^{*}\left(c+E_{0} x_{0}\right)^{2}\left[\left(b c E_{0}+3 b c R x_{0}\right)-e R\left(c+E_{0} x_{0}\right)^{2}\right]}{\left[e\left(c+E_{0} x_{0}\right)^{2}-b c x_{0}\right]^{2}}\right] \\
& +\bar{z}^{2}\left[\frac{a m \bar{q}_{2}^{*} y_{0}}{\left(a+x_{0}\right)^{3}}-\frac{a m \overline{q_{2}} \overline{q_{2}^{*}}}{\left(a+x_{0}\right)^{2}}-R \overline{q_{2}^{*}}-\frac{r \overline{q_{2}^{*}}}{K} e^{2 i \omega \tau_{n} \theta}+\frac{a c m \overline{q_{2}}}{\left(a+x_{0}\right)^{2}}-\frac{a c m y_{0}}{\left(a+x_{0}\right)^{3}}\right. \\
& +\frac{b c e q_{2}^{*} x_{0} E_{0}\left(c+E_{0} x_{0}\right)\left(E_{0}+R x_{0}\right)+b^{2} c^{2} R \bar{q}_{2}^{*} x_{0}^{2}}{\left[e\left(c+E_{0} x_{0}\right)^{2}-b c x_{0}\right]^{2}} \\
& \left.-\frac{e q_{2}^{*}\left(c+E_{0} x_{0}\right)^{2}\left[\left(b c E_{0}+3 b c R x_{0}\right)-e R\left(c+E_{0} x_{0}\right)^{2}\right]}{2\left[e\left(c+E_{0} x_{0}\right)^{2}-b c x_{0}\right]^{2}}\right] \\
& +z^{2} \bar{z}\left[\left(\frac{2 a m q_{2}^{*} y_{0}}{\left(a+x_{0}\right)^{3}}-\frac{a m q_{2} \bar{q}_{2}^{*}}{\left(a+x_{0}\right)^{2}}-2 R \overline{q_{2}^{*}}+\frac{a c m q_{2}}{\left(a+x_{0}\right)^{2}}-\frac{2 a c m y_{0}}{\left(a+x_{0}\right)^{3}}\right.\right. \\
& +\frac{2 b c e q_{2}^{*} x_{0} E_{0}\left(c+E_{0} x_{0}\right)\left(E_{0}+R x_{0}\right)+2 b^{2} c^{2} R q_{2}^{*} x_{0}^{2}}{\left[e\left(c+E_{0} x_{0}\right)^{2}-b c x_{0}\right]^{2}} \\
& \left.-\frac{e \bar{q}_{2}^{*}\left(c+E_{0} x_{0}\right)^{2}\left[\left(b c E_{0}+3 b c R x_{0}\right)-e R\left(c+E_{0} x_{0}\right)^{2}\right]}{\left[e\left(c+E_{0} x_{0}\right)^{2}-b c x_{0}\right]^{2}}\right) W_{11}^{(1)}(0) \\
& +\left(\frac{a c m}{\left(a+x_{0}\right)^{2}}-\frac{a m q_{2}^{*}}{\left(a+x_{0}\right)^{2}}\right) W_{11}^{(2)}(0)
\end{aligned}
$$




$$
\begin{aligned}
& +\left(\frac{a m \overline{q_{2}^{*} y_{0}}}{\left(a+x_{0}\right)^{3}}-\frac{a m \overline{q_{2}} \bar{q}_{2}^{*}}{2\left(a+x_{0}\right)^{2}}-R \overline{q_{2}^{*}}+\frac{a c m \overline{q_{2}}}{2\left(a+x_{0}\right)^{2}}-\frac{a c m y_{0}}{\left(a+x_{0}\right)^{3}}\right. \\
& \left.+\frac{b c e \overline{q_{2}^{*}} x_{0} E_{0}\left(c+E_{0} x_{0}\right)\left(E_{0}+R x_{0}\right)+b^{2} c^{2} R \overline{q_{2}^{*} x_{0}^{2}}}{\left[e\left(c+E_{0} x_{0}\right)^{2}-b c x_{0}\right]^{2}}\right) W_{20}^{(1)}(0) \\
& -\frac{e \overline{q_{2}^{*}}\left(c+E_{0} x_{0}\right)^{2}\left[\left(b c E_{0}+3 b c R x_{0}\right)-e R\left(c+E_{0} x_{0}\right)^{2}\right]}{2\left[e\left(c+E_{0} x_{0}\right)^{2}-b c x_{0}\right]^{2}} \\
& +\left(\frac{a c m}{2\left(a+x_{0}\right)^{2}}-\frac{a m \bar{q}_{2}^{*}}{2\left(a+x_{0}\right)^{2}}\right) W_{20}^{(2)}(0) \\
& \left.\left.-\frac{2 r q_{2}^{*}}{K} e^{-i \omega \tau_{n} \theta} W_{11}^{(1)}(-1)-\frac{r q_{2}^{*}}{K} e^{i \omega \tau_{n} \theta} W_{20}^{(1)}(-1)\right]+\cdots\right\} .
\end{aligned}
$$

Equating the corresponding coefficients in (3.14) and (3.15) leads to the relations

$$
\begin{aligned}
& g_{20}=\frac{2 \tau_{n}}{\bar{D}}\left[\frac{a m \bar{q}_{2}^{*} y_{0}}{\left(a+x_{0}\right)^{3}}-\frac{a m q_{2} \bar{q}_{2}^{*}}{\left(a+x_{0}\right)^{2}}-R \bar{q}_{2}^{*}-\frac{r \bar{q}_{2}^{*}}{K} e^{-2 i \omega \tau_{n} \theta}+\frac{a c m q_{2}}{\left(a+x_{0}\right)^{2}}-\frac{a c m y_{0}}{\left(a+x_{0}\right)^{3}}\right. \\
& +\frac{b c e q_{2}^{*} x_{0} E_{0}\left(c+E_{0} x_{0}\right)\left(E_{0}+R x_{0}\right)+b^{2} c^{2} R \bar{q}_{2}^{*} x_{0}^{2}}{\left[e\left(c+E_{0} x_{0}\right)^{2}-b c x_{0}\right]^{2}} \\
& \left.-\frac{e q_{2}^{*}\left(c+E_{0} x_{0}\right)^{2}\left[\left(b c E_{0}+3 b c R x_{0}\right)-e R\left(c+E_{0} x_{0}\right)^{2}\right]}{2\left[e\left(c+E_{0} x_{0}\right)^{2}-b c x_{0}\right]^{2}}\right] \text {, } \\
& g_{11}=\frac{\tau_{n}}{\bar{D}}\left[\frac{2 a m \bar{q}_{2}^{*} y_{0}}{\left(a+x_{0}\right)^{3}}-\frac{2 a m \bar{q}_{2}^{*} \operatorname{Re}\left(q_{2}\right)}{\left(a+x_{0}\right)^{2}}-2 R \overline{q_{2}^{*}}-\frac{2 r \bar{q}_{2}^{*}}{K}+\frac{2 a c m \operatorname{Re}\left(q_{2}\right)}{\left(a+x_{0}\right)^{2}}-\frac{2 a c m y_{0}}{\left(a+x_{0}\right)^{3}}\right. \\
& +\frac{2 b c e q_{2}^{*} x_{0} E_{0}\left(c+E_{0} x_{0}\right)\left(E_{0}+R x_{0}\right)+2 b^{2} c^{2} R \bar{q}_{2}^{*} x_{0}^{2}}{\left[e\left(c+E_{0} x_{0}\right)^{2}-b c x_{0}\right]^{2}} \\
& \left.-\frac{e q_{2}^{*}\left(c+E_{0} x_{0}\right)^{2}\left[\left(b c E_{0}+3 b c R x_{0}\right)-e R\left(c+E_{0} x_{0}\right)^{2}\right]}{\left[e\left(c+E_{0} x_{0}\right)^{2}-b c x_{0}\right]^{2}}\right] \text {, } \\
& g_{02}=\frac{2 \tau_{n}}{\bar{D}}\left[\frac{a m \overline{q_{2}^{*}} y_{0}}{\left(a+x_{0}\right)^{3}}-\frac{a m \overline{q_{2}} \overline{q_{2}^{*}}}{\left(a+x_{0}\right)^{2}}-R \overline{q_{2}^{*}}-\frac{r \overline{q_{2}^{*}}}{K} e^{2 i \omega \tau_{n} \theta}+\frac{a c m \overline{q_{2}}}{\left(a+x_{0}\right)^{2}}-\frac{a c m y_{0}}{\left(a+x_{0}\right)^{3}}\right. \\
& +\frac{b c e q_{2}^{*} x_{0} E_{0}\left(c+E_{0} x_{0}\right)\left(E_{0}+R x_{0}\right)+b^{2} c^{2} R \overline{q_{2}^{*} x_{0}^{2}}}{\left[e\left(c+E_{0} x_{0}\right)^{2}-b c x_{0}\right]^{2}} \\
& \left.-\frac{e q_{2}^{*}\left(c+E_{0} x_{0}\right)^{2}\left[\left(b c E_{0}+3 b c R x_{0}\right)-e R\left(c+E_{0} x_{0}\right)^{2}\right]}{2\left[e\left(c+E_{0} x_{0}\right)^{2}-b c x_{0}\right]^{2}}\right] \text {, } \\
& g_{21}=\frac{2 \tau_{n}}{\bar{D}}\left[\left(\frac{2 a m q_{2}^{*} y_{0}}{\left(a+x_{0}\right)^{3}}-\frac{a m q_{2} \bar{q}_{2}^{*}}{\left(a+x_{0}\right)^{2}}-2 R \bar{q}_{2}^{*}+\frac{a c m q_{2}}{\left(a+x_{0}\right)^{2}}-\frac{2 a c m y_{0}}{\left(a+x_{0}\right)^{3}}\right.\right. \\
& +\frac{2 b c e q_{2}^{*} x_{0} E_{0}\left(c+E_{0} x_{0}\right)\left(E_{0}+R x_{0}\right)+2 b^{2} c^{2} R \bar{q}_{2}^{*} x_{0}^{2}}{\left[e\left(c+E_{0} x_{0}\right)^{2}-b c x_{0}\right]^{2}}
\end{aligned}
$$


Bifurcation in a Differential-Algebra Predator-Prey System with Time Lag Effects

$$
\begin{aligned}
& \left.-\frac{e \overline{q_{2}^{*}}\left(c+E_{0} x_{0}\right)^{2}\left[\left(b c E_{0}+3 b c R x_{0}\right)-e R\left(c+E_{0} x_{0}\right)^{2}\right]}{\left[e\left(c+E_{0} x_{0}\right)^{2}-b c x_{0}\right]^{2}}\right) W_{11}^{(1)}(0) \\
& +\left(\frac{a c m}{\left(a+x_{0}\right)^{2}}-\frac{a m \overline{q_{2}^{*}}}{\left(a+x_{0}\right)^{2}}\right) W_{11}^{(2)}(0) \\
& +\left(\frac{a m q_{2}^{*} y_{0}}{\left(a+x_{0}\right)^{3}}-\frac{a m \overline{q_{2}} \bar{q}_{2}^{*}}{2\left(a+x_{0}\right)^{2}}-R \overline{q_{2}^{*}}+\frac{a c m \overline{q_{2}}}{2\left(a+x_{0}\right)^{2}}-\frac{a c m y_{0}}{\left(a+x_{0}\right)^{3}}\right. \\
& \left.+\frac{b c e \overline{q_{2}^{*}} x_{0} E_{0}\left(c+E_{0} x_{0}\right)\left(E_{0}+R x_{0}\right)+b^{2} c^{2} R \bar{q}_{2}^{*} x_{0}^{2}}{\left[e\left(c+E_{0} x_{0}\right)^{2}-b c x_{0}\right]^{2}}\right) W_{20}^{(1)}(0) \\
& -\frac{e q_{2}^{*}\left(c+E_{0} x_{0}\right)^{2}\left[\left(b c E_{0}+3 b c R x_{0}\right)-e R\left(c+E_{0} x_{0}\right)^{2}\right]}{2\left[e\left(c+E_{0} x_{0}\right)^{2}-b c x_{0}\right]^{2}} \\
& +\left(\frac{a c m}{2\left(a+x_{0}\right)^{2}}-\frac{a m q_{2}^{*}}{2\left(a+x_{0}\right)^{2}}\right) W_{20}^{(2)}(0) \\
& \left.-\frac{2 r q_{2}^{*}}{K} e^{-i \omega \tau_{n} \theta} W_{11}^{(1)}(-1)-\frac{r q_{2}^{*}}{K} e^{i \omega \tau_{n} \theta} W_{20}^{(1)}(-1)\right] .
\end{aligned}
$$

We note that the coefficients $g_{20}, g_{11}$ and $g_{02}$ are well defined. On the other hand, $W_{20}(\theta)$ and $W_{11}(\theta)$ in the expression for $g_{21}$ are not yet determined. To find them, we use the approach of [18], thus obtaining

$$
\begin{aligned}
& W_{20}(\theta)=\frac{i g_{20}}{\omega \tau_{n}} q(0) e^{i \omega \tau_{n} \theta}+\frac{i \bar{g}_{02}}{3 \omega \tau_{n}} \bar{q}(0) e^{-i \omega \tau_{n} \theta}+\mathscr{E}_{1} e^{2 i \omega \tau_{n} \theta}, \\
& W_{11}(\theta)=-\frac{i g_{11}}{\omega \tau_{n}} q(0) e^{i \omega \tau_{n} \theta}+\frac{i \bar{g}_{11}}{\omega \tau_{n}} \bar{q}(0) e^{-i \omega \tau_{n} \theta}+\mathscr{E}_{2},
\end{aligned}
$$

where

$$
\begin{gathered}
\mathscr{E}_{1}=2\left(\begin{array}{cc}
2 i \omega+R x_{0}+\frac{b c x_{0}\left(E_{0}+R x_{0}\right)-e R x_{0}\left(c+E_{0} x_{0}\right)^{2}}{e\left(c+E_{0} x_{0}\right)^{2}-b c x_{0}} & m x_{0} \\
-\frac{m x_{0} y_{0}}{\left(a+x_{0}\right)^{2}}+\frac{r}{K} x_{0} e^{-2 i \omega \tau_{n}} & 2 i \omega \\
-\frac{a c m y_{0}}{\left(a+x_{0}\right)^{2}} &
\end{array}\right)^{-1}\left(\begin{array}{l}
\mathscr{P}_{11} \\
\mathscr{P}_{21}
\end{array}\right), \\
\mathscr{E}_{2}=2\left(\begin{array}{cc}
R x_{0}+\frac{b c x_{0}\left(E_{0}+R x_{0}\right)-e R x_{0}\left(c+E_{0} x_{0}\right)^{2}}{e\left(c+E_{0} x_{0}\right)^{2}-b c x_{0}} \\
-\frac{m x_{0} y_{0}}{\left(a+x_{0}\right)^{2}}+\frac{r}{K} x_{0} \\
-\frac{a c m y_{0}}{\left(a+x_{0}\right)^{2}} & 0
\end{array}\right)^{-1}\left(\begin{array}{l}
\mathscr{Q}_{11} \\
\mathscr{Q}_{21}
\end{array}\right),
\end{gathered}
$$

and

$$
\mathscr{P}_{11}=\frac{a m y_{0}}{\left(a+x_{0}\right)^{3}}-R+\frac{b c e x_{0} E_{0}\left(c+E_{0} x_{0}\right)\left(E_{0}+R x_{0}\right)+R b^{2} c^{2} x_{0}^{2}}{\left[e\left(c+E_{0} x_{0}\right)^{2}-b c x_{0}\right]^{2}}
$$




$$
\begin{aligned}
& -\frac{e\left(c+E_{0} x_{0}\right)^{2}\left[\left(b c E_{0}+3 b c R x_{0}\right)-e R\left(c+E_{0} x_{0}\right)^{2}\right]}{2\left[e\left(c+E_{0} x_{0}\right)^{2}-b c x_{0}\right]^{2}} \\
& -\frac{a m q_{2}}{\left(a+x_{0}\right)^{2}}-\frac{r}{K} e^{-2 i \omega \tau_{n}}, \\
\mathscr{P}_{21}= & \frac{a c m q_{2}}{\left(a+x_{0}\right)^{2}}-\frac{a c m y_{0}}{\left(a+x_{0}\right)^{3}}, \\
\mathscr{Q}_{11}= & \frac{a m y_{0}}{\left(a+x_{0}\right)^{3}}-R+\frac{b c e x_{0} E_{0}\left(c+E_{0} x_{0}\right)\left(E_{0}+R x_{0}\right)+R b^{2} c^{2} x_{0}^{2}}{\left[e\left(c+E_{0} x_{0}\right)^{2}-b c x_{0}\right]^{2}} \\
& -\frac{e\left(c+E_{0} x_{0}\right)^{2}\left[\left(b c E_{0}+3 b c R x_{0}\right)-e R\left(c+E_{0} x_{0}\right)^{2}\right]}{2\left[e\left(c+E_{0} x_{0}\right)^{2}-b c x_{0}\right]^{2}} \\
& -\frac{a m \operatorname{Re}\left(q_{2}\right)}{\left(a+x_{0}\right)^{2}}-\frac{r}{K}, \\
\mathscr{Q}_{21}= & \frac{a c m \operatorname{Re}\left(q_{2}\right)}{\left(a+x_{0}\right)^{2}}-\frac{a c m y_{0}}{\left(a+x_{0}\right)^{3}} .
\end{aligned}
$$

Immediate calculations show that

$$
\mathscr{E}_{1}=\left(\begin{array}{c}
\frac{4 \mathscr{P}_{11} i \omega-\frac{2 m x_{0}}{a+x_{0}} \mathscr{P}_{21}}{\Upsilon_{1}} \\
\frac{2 a c m y_{0}}{\left(a+x_{0}\right)^{2}} \mathscr{P}_{11}+\Upsilon_{2} \mathscr{P}_{21} \\
\Upsilon_{1}
\end{array}\right)_{2 \times 1}, \quad \mathscr{E}_{2}=\left(\begin{array}{c}
-\frac{2\left(a+x_{0}\right)^{2}}{a c m y_{0}} \mathscr{Q}_{21} \\
\frac{2\left(a+x_{0}\right)}{m x_{0}} \mathscr{Q}_{11}-2 \Upsilon_{3} \mathscr{Q}_{21}
\end{array}\right)_{2 \times 1}
$$

where

$$
\begin{aligned}
\Upsilon_{1}= & \frac{a c m^{2} x_{0} y_{0}}{\left(a+x_{0}\right)^{3}}+\frac{2 r}{K} x_{0} i \omega e^{-2 i \omega \tau_{n}}-4 \omega^{2} \\
& -\left(\frac{2 m x_{0} y_{0}}{\left(a+x_{0}\right)^{2}}-2 R x_{0}-\frac{2 b c x_{0}\left(E_{0}+R x_{0}\right)-2 e R x_{0}\left(c+E_{0} x_{0}\right)^{2}}{e\left(c+E_{0} x_{0}\right)^{2}-b c x_{0}}\right) i \omega, \\
\Upsilon_{2}= & 4 i \omega+2 R x_{0}+\frac{2 b c x_{0}\left(E_{0}+R x_{0}\right)-2 e R x_{0}\left(c+E_{0} x_{0}\right)^{2}}{e\left(c+E_{0} x_{0}\right)^{2}-b c x_{0}} \\
& -\frac{2 m x_{0} y_{0}}{\left(a+x_{0}\right)^{2}}+\frac{2 r}{K} x_{0} e^{-2 i \omega \tau_{n}}, \\
\Upsilon_{3}= & \frac{a+x_{0}}{a c m}-\frac{R\left(a+x_{0}\right)^{3}}{a c m^{2} y_{0}}-\frac{\left(a+x_{0}\right)^{3}\left[b c\left(E_{0}+R x_{0}\right)-e R\left(c+E_{0} x_{0}\right)^{2}\right]}{a c m^{2} y_{0}\left[e\left(c+E_{0} x_{0}\right)^{2}-b c x_{0}\right]}-\frac{r\left(a+x_{0}\right)^{3}}{a c m^{2} K y_{0}} .
\end{aligned}
$$

Substituting $\mathscr{E}_{1}$ and $\mathscr{E}_{2}$ into the Eqs. (3.16), we obtain the terms $W_{20}(\theta)$ and $W_{11}(\theta)$ and $g_{21}$. Therefore, the critical parameters 


$$
\begin{aligned}
& c_{1}(0)=\frac{i}{2 \omega \tau_{n}}\left(g_{11} g_{20}-2\left|g_{11}\right|^{2}-\frac{\left|g_{02}\right|^{2}}{3}\right)+\frac{g_{21}}{2}, \\
& \mu_{2}=-\frac{\operatorname{Re}\left\{c_{1}(0)\right\}}{\operatorname{Re}\left\{\lambda^{\prime}\left(\tau_{n}\right)\right\}}, \quad \beta_{2}=2 \operatorname{Re}\left\{c_{1}(0)\right\}, \\
& T_{2}=-\frac{\operatorname{Im}\left\{c_{1}(0)\right\}+\mu_{2} \operatorname{Im}\left\{\lambda^{\prime}\left(\tau_{n}\right)\right\}}{\omega \tau_{n}}
\end{aligned}
$$

defining the properties of Hopf bifurcation for $\tau=\tau_{n}$ are also known $-\mathrm{cf}$. [18].

Using the necessary conditions for Hopf bifurcations in the differential-algebra predator-prey system (1.5), we arrive at the following result connected with the classical Hopf bifurcation theorem [18].

Theorem 3.1. Assume that the system (1.5) has a positive time lag $\tau$ and

$$
\begin{aligned}
& \frac{r}{K}+R>\frac{m y_{0}}{\left(a+x_{0}\right)^{2}}, \\
& {\left[x_{0}\left(\frac{m y_{0}}{\left(a+x_{0}\right)^{2}}-R\right)^{2}-\frac{r^{2}}{K^{2}} x_{0}-\frac{2 a c m^{2} y_{0}}{\left(a+x_{0}\right)^{3}}\right]^{2}>\frac{4 a^{2} c^{2} m^{4} y_{0}^{2}}{\left(a+x_{0}\right)^{6}}} \\
& x_{0}\left(\frac{m y_{0}}{\left(a+x_{0}\right)^{2}}-R\right)^{2}<\frac{r^{2}}{K^{2}} x_{0}+\frac{2 a c m^{2} y_{0}}{\left(a+x_{0}\right)^{3}} .
\end{aligned}
$$

Then a Hopf bifurcation can only occur at a positive equilibrium $X_{0}$ if the time lag $\tau$ is one of the bifurcation value $\tau_{n}^{ \pm}, n=0,1,2, \cdots, N$. Moreover,

(i) If $\mu_{2}>0\left(\mu_{2}<0\right)$, then the Hopf bifurcation is supercritical (subcritical).

(ii) If $\beta_{2}<0\left(\beta_{2}>0\right)$, then the bifurcating periodic orbits are stable (unstable).

(iii) If $T_{2}>0\left(T_{2}<0\right)$, then the period of the bifurcating periodic orbits increases (decreases).

\section{Example}

Let us consider the system (1.5) with parameters $r=6, K=3 / 2, m=1, a=1, d=$ $1 / 2, c=1, b=6, e=1$ and $v=2-$ i.e.

$$
\begin{aligned}
& \dot{x}(t)=x(t)\left(6-4 x(t-\tau)-\frac{y(t)}{1+x(t)}-E(t)\right), \\
& \dot{y}(t)=y(t)\left(-\frac{1}{2}+\frac{x(t)}{1+x(t)}\right) \\
& 0=E(t) x(t)\left(\frac{6}{1+E(t) x(t)}-\frac{1}{x(t)}\right)-2 .
\end{aligned}
$$


The coefficients of (4.1) satisfy the assumptions (2.1) and simple computations shows that this system has a positive equilibrium $X_{0}^{*}=(1,2,1)$ and $R=-3$. It follows that

$$
\begin{aligned}
& (r / K)+R=1>m y_{0} /\left(a+x_{0}\right)^{2}=0.5 \\
& \left.x_{0}\left[m y_{0} /\left(a+x_{0}\right)^{2}-R\right]^{2}-\left(r^{2} / K^{2}\right) x_{0}-2 a c m^{2} y_{0} /\left(a+x_{0}\right)^{3}\right\}^{2} \\
= & 18.0625>4 a^{2} c^{2} m^{4} y_{0}^{2} /\left(a+x_{0}\right)^{6}=0.25 \\
& x_{0}\left[m y_{0} /\left(a+x_{0}\right)^{2}-R\right]^{2}=12.25<\left(r^{2} / K^{2}\right) x_{0}+2 a c m^{2} y_{0} /\left(a+x_{0}\right)^{3}=16.5 .
\end{aligned}
$$

Hence, this system satisfies the conditions for occurrence Hopf bifurcation - cf. Theorems 2.1 and 3.1.

The corresponding equation (2.11) takes the form $\omega^{4}-(17 / 4) \omega^{2}+(1 / 16)=0$. It has two positive roots $\omega^{+}=2.0580$ and $\omega^{-}=0.1215$ derived with the assistance of MATLAB 7.0. Therefore, $\tau_{0}^{+}=(1 / 2.0580) \arccos (7 / 8)=0.2456, \tau_{0}^{-}=(1 / 0.1215) \arccos (7 / 8)$ $=4.1593$ and the terms in (3.17) are also defined - viz.

$$
\begin{aligned}
& c_{1}(0)=1.1374-9.5281 i, \quad \lambda^{\prime}\left(\tau_{0}^{+}\right)=15.8647-1.7309 i, \\
& \mu_{2}=-0.0717<0, \quad \beta_{2}=2.2748>0, \quad T_{2}=18.6054>0 .
\end{aligned}
$$

Theorem 2.1 yields that if $\tau \in[0,0.2456)$, then the equilibrium $X_{0}^{*}(1,2,1)$ is locally asymptotically stable and it is unstable if $\tau \in(0.2456,4.1593)$. Thus a Hopf bifurcation occurs at the equilibrium $X_{0}^{*}=(1,2,1)$ for the time lag $\tau_{0}^{+}=0.2456$. By Theorem 3.1, the Hopf bifurcation at $\tau_{0}^{+}$is subcritical and the corresponding bifurcating periodic orbits increase and are unstable.

To verify the above conclusions, we present numerical results for the example (4.1) at different time lags $\tau$. MATLAB simulations show that the equilibrium $X_{0}^{*}(1,2,1)$ is locally asymptotically stable if $\tau=0$ and $\tau=0.243<\tau_{0}^{+}-$cf. Figs. 1-2. If $\tau$ takes the bifurcation value $\tau_{0}^{+}=0.2456$, then periodic orbits bifurcate from the equilibrium $X_{0}^{*}(1,2,1)-$ cf. Fig. 3. If $\tau=0.24563>\tau_{0}^{+}$, the periodic orbits are bifurcating from the equilibrium $X_{0}^{*}(1,2,1)$ are unstable and increase - cf. Fig. 4 , and if $\tau=0.2485>\tau_{0}^{+}$, the equilibrium $X_{0}^{*}(1,2,1)$ is unstable - cf. Fig. 5 .

\section{Discussion}

The understanding of population dynamics in predator-prey systems can be very helpful in the study of multiple species interactions in biological systems [9, 14, 26, 37]. In this work, we considered the impact of time lag on the population dynamics in the differentialalgebra predator-prey system (1.5) and observed that the presence of time lag makes the stability and bifurcation analysis much more complicated. Our study shows that the positive equilibrium of the predator-prey system is locally asymptotically stable for sufficiently small time lags $\tau \geq 0$. If $\tau$ grows, then for the sequence of Hopf bifurcation values, the stability of a positive equilibrium switches back and force several times and the equilibrium becomes unstable for sufficiently large $\tau$. Stable equilibrium ensures that the populations 

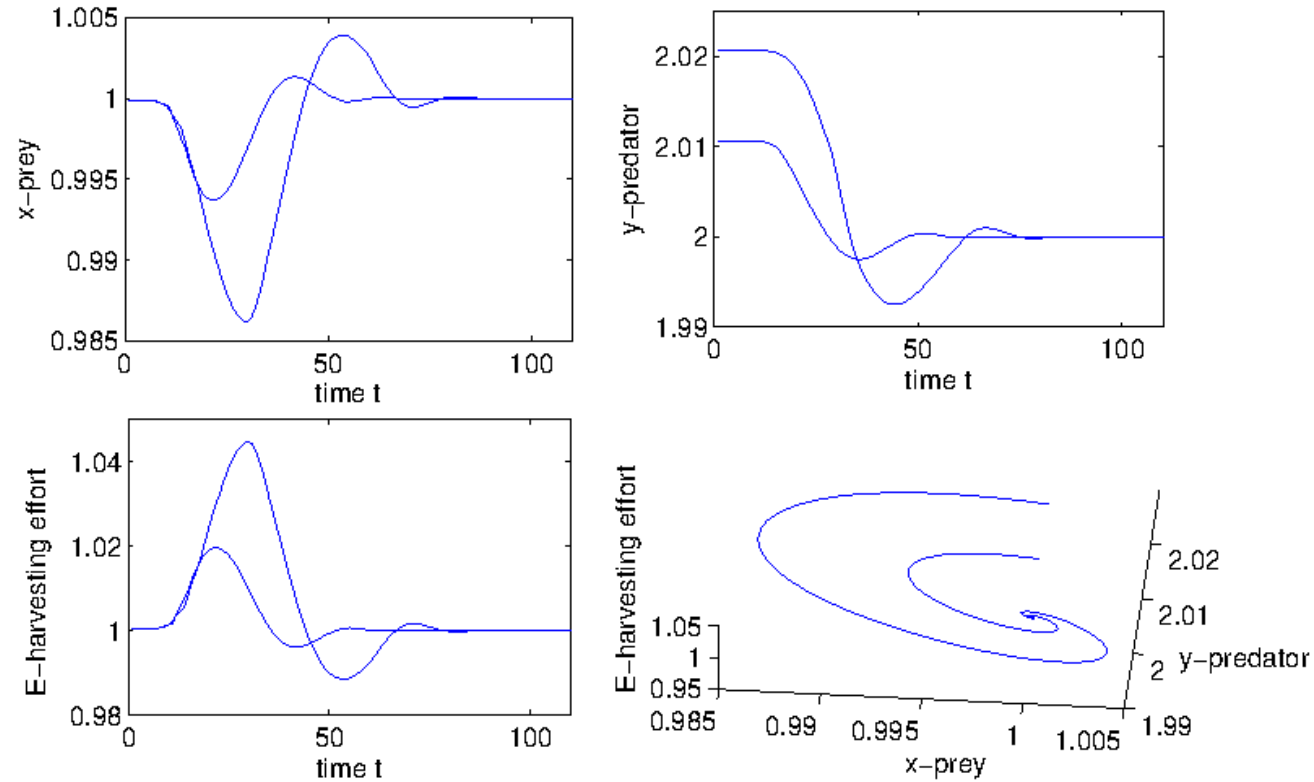

Figure 1: Positive equilibrium $X_{0}^{*}(1,2,1)$ is locally asymptotically stable, $\tau=0$. Initial values are $(x(0), y(0), E(0))=(0.9999,2.0006+0.01 * \mathrm{~m}, 0.9999), \mathrm{m}=1,2$.
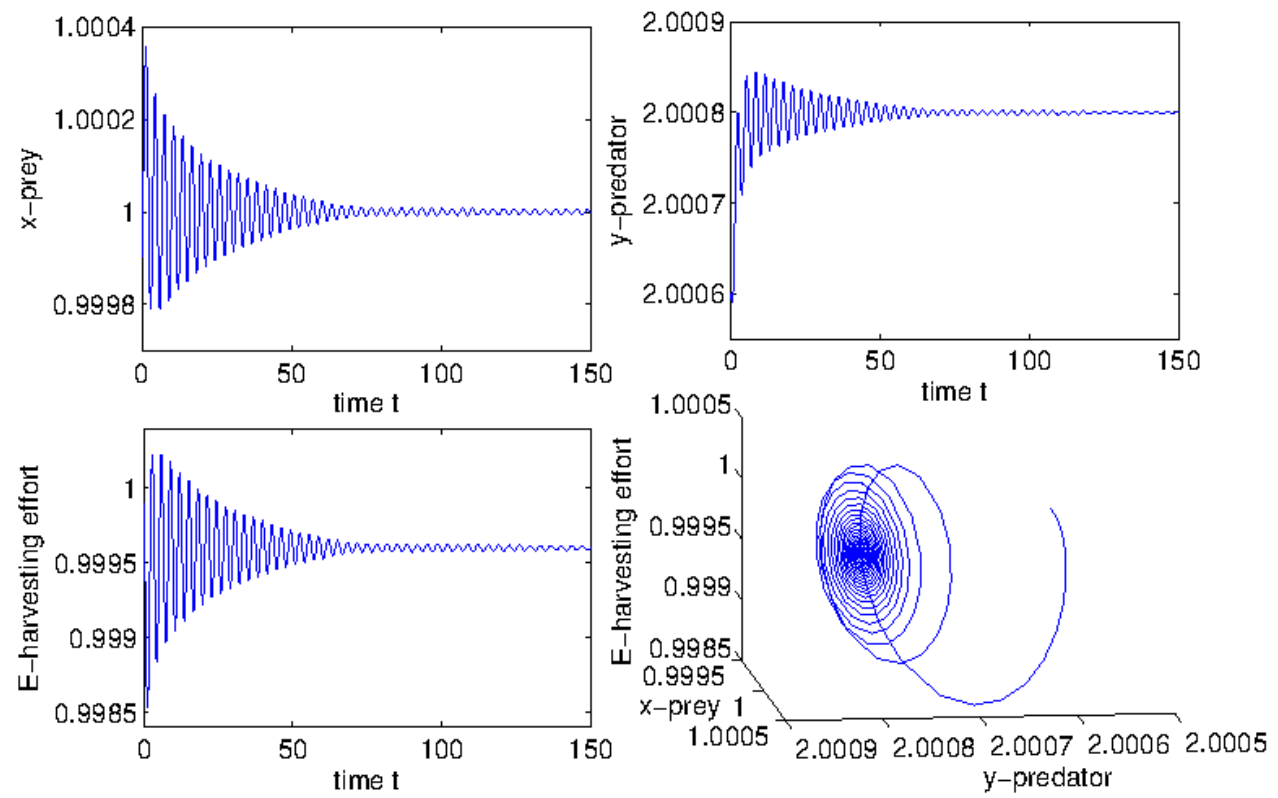

Figure 2: Positive equilibrium $X_{0}^{*}(1,2,1)$ is locally asymptotically stable, $\tau=0.243<\tau_{0}^{+}=0.2456$. Initial values are $(x(\theta), y(0), E(0))=(0.9999,2.0006,0.9999)$. 

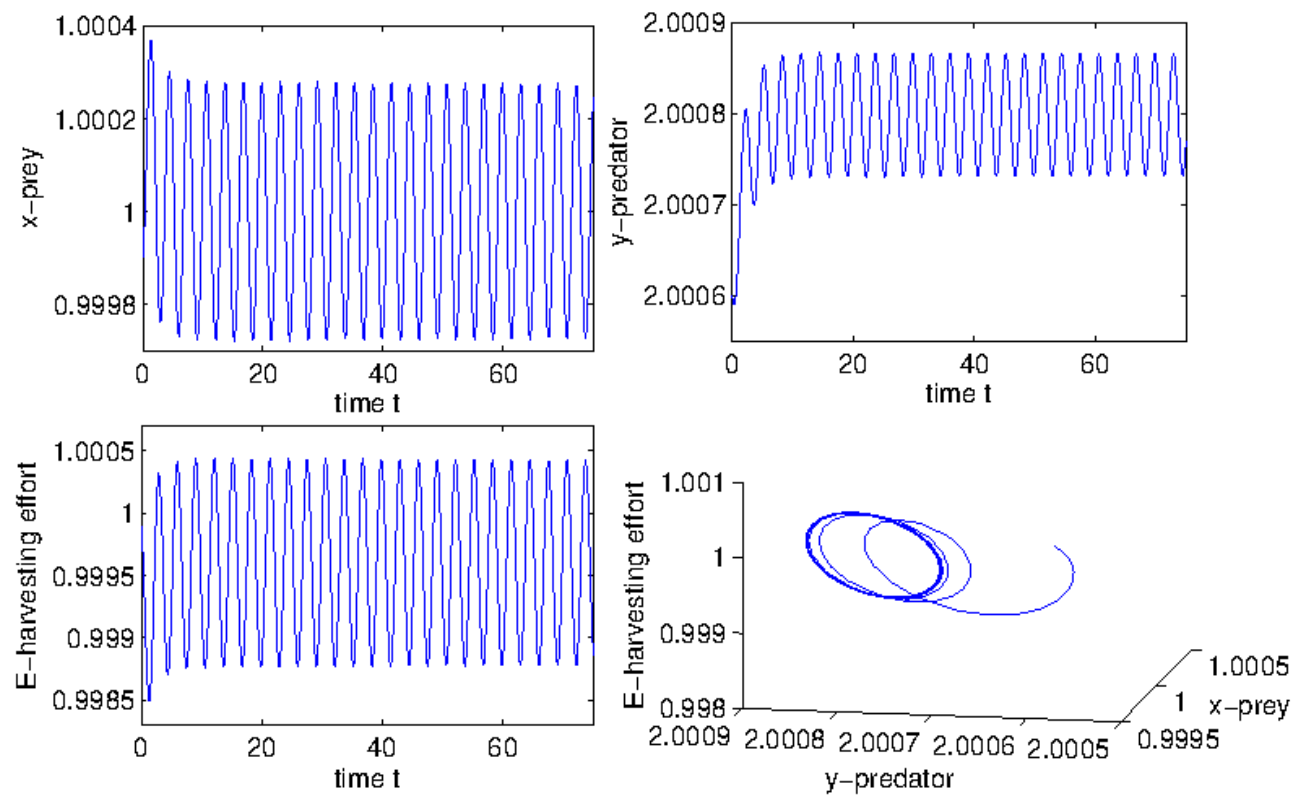

Figure 3: Periodic orbits bifurcate from the positive equilibrium $X_{0}^{*}(1,2,1), \tau_{0}^{+}=0.2456$. Initial values are $(x(\theta), y(0), E(0))=(0.9999,2.0006,0.9999)$.
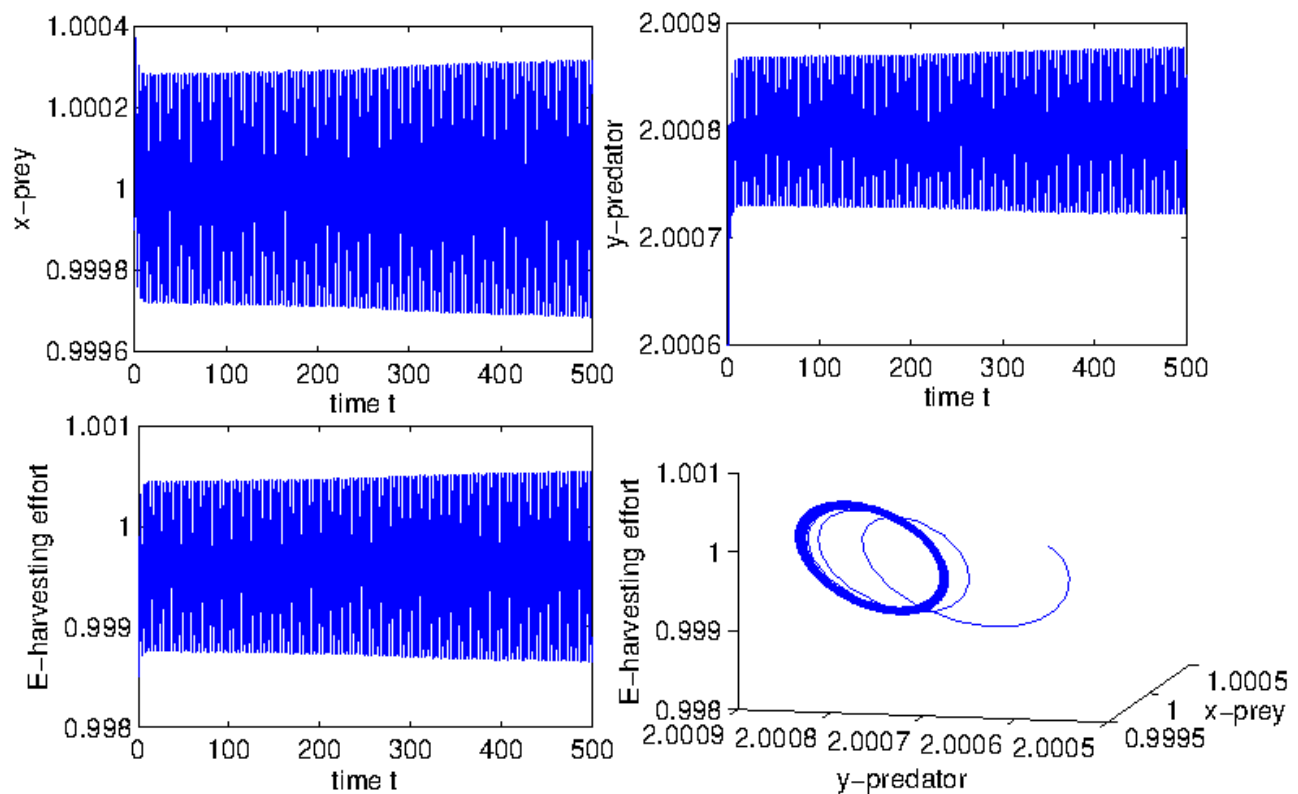

Figure 4: Periodic orbits bifurcating from the positive equilibrium $X_{0}^{*}(1,2,1)$ increase and are unstable, $\tau=0.24563>\tau_{0}^{+}=0.2456$. Initial values are $(x(\theta), y(0), E(0))=(0.9999,2.0006,0.9999)$. 

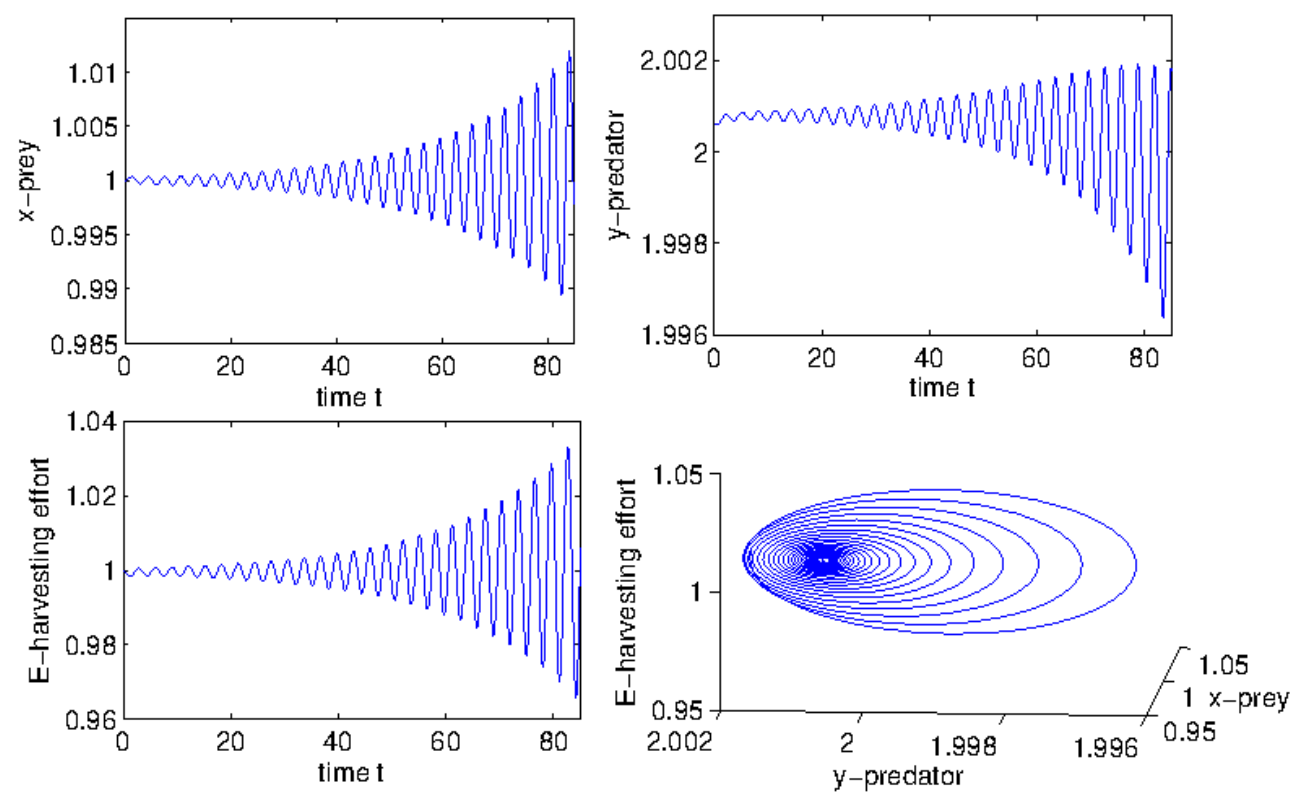

Figure 5: Positive equilibrium $X_{0}^{*}(1,2,1)$ is unstable, $\tau=0.2485>\tau_{0}^{+}=0.2456$. Initial values are $(x(\theta), y(0), E(0))=(0.9999,2.0006,0.9999)$.

of predators and preys coexist and arrive at a natural balance state as time $t$ goes on. Consequently, the sustainability of such differential-algebra predator-prey system is protected. However, if a positive equilibrium is unstable, then the predator-prey system can easily lose its ecological balance under small disturbance by the outside world, with possible disappearance of both predator and prey species.

On the other hand, the stability switches of a positive equilibrium would generate families of small-amplitude periodic orbits bifurcating from the equilibrium. The appearance of bifurcating periodic orbits would result in small-amplitude oscillations of population densities in our predator-prey system. This can be considered as the periodic evolution of prey and predator species. The stable periodic orbits indicate that the populations of predators and preys with the initial population densities (1.6) (around the positive equilibrium values) can still coexist in an oscillatory mode. However, unstable periodic orbits produce the growing oscillations of populations which can cause the extinction of the ecosystem. Therefore, the study of the stability, direction, and periodic orbits arising in Hopf bifurcations play an important role in the understanding of oscillatory actions in the differentialalgebra predator-prey system (1.5).

We also note that the external harvesting effort $E(t)$ may not be unique. If we consider the system (1.5) from the perspective of differential equations - i.e. if we derive $E(t)$ from the Eq. (1.5) and substitute it into the first differential equation of (1.5), then the following two cases have to be discussed:

(i) $\triangle=[c e+v x(t)-b x(t)]^{2}-4 c v e x(t)>0$ and (1.5) has two positive real roots $E_{1,2}(t)$. 
(ii) $\Delta=[c e+v x(t)-b x(t)]^{2}-4 c v e x(t)=0$ and (1.5) has only one positive root $E_{1}(t)$.

These cases are connected with the delayed differential subsystems, all of which should be studied if we want to have dynamical results for the original system (1.5). However, as was seen in Section 3, the use of the center manifold theorem is a very complicated matter even for one delayed differential system (3.8). Therefore, working with $3 \times 3$ delayed differential systems lead to big computational problems and here, the system (1.5) is studied as a whole, without extracting the variable $E(t)$. In fact, differential-algebra equations are different from differential equations - cf. Refs. [4, 21-23, 34]. In Section 2, we apply the parametrisation method for differential-algebra equations to study the dynamics of (1.5), thus reducing the initial system (1.5) to a 2-dimensional delayed differential system (3.8). Hence, the local stability and Hopf bifurcations of the original system (1.5) can be determined only by the delayed differential system (3.8). This approach is much more convenient than direct conversion into three delayed differential subsystems.

The approach of this work can be used in other problems, such as

(i) Since predators require a time to go to the maturity stage $[14,26]$, the corresponding maturation time lag and the combined impacts of the double time lag on the population dynamics can be incorporated into the system (1.5).

(ii) Stage-structure of biological population, which is extremely important in population dynamical systems can be included into our predator-prey model $-\mathrm{cf}$. [2,11].

(iii) The discrete-time dynamical systems are of vital significance in the field of population biology $[8,9]$ and can be studied within corresponding discrete systems for (1.5).

\section{Acknowledgments}

The authors are greatly indebted to the anonymous referees and the editor for care-

ful reading and valuable suggestions, which greatly improved the quality of the original manuscript.

This work is supported by the National Natural Science Foundations of China (Grants Nos. 11871393, 61663043), the Science and Technology Project founded by the Education Department of Jiangxi Province (Grant No. GJJ14775), the International Science and Technology Cooperation Program of Shaanxi Key Research \& Development Plan (Grant No. S2019-YF-GHZD-0003).

\section{A. Appendix}

Here we provide a short introduction to the parametrisation method [7]. Consider the following DAE system:

$$
\begin{aligned}
& \dot{\tilde{X}}(t)=f(\tilde{X}(t)), \\
& 0=g(\tilde{X}(t)),
\end{aligned}
$$


where $f: \mathrm{R}^{n} \rightarrow \mathrm{R}^{n}, g: \mathrm{R}^{n} \rightarrow \mathrm{R}^{\mathbb{k}}\left(\mathbb{k}_{k}<n\right)$ are continuous differentiable functions, $\widetilde{X}(t)=$ $\left(\widetilde{x}_{1}(t), \widetilde{x}_{2}(t), \cdots, \widetilde{x}_{n}(t)\right)^{T} \in \mathrm{R}^{n}, f=\left(f_{1}, f_{2}, \cdots, f_{n}\right)^{T}, g=\left(g_{1}, g_{2}, \cdots, g_{\mathbb{k}}\right)^{T}$. We also assume that DAE system (A.1) has an equilibrium $\tilde{X}_{0}$.

If $\operatorname{rank} D_{\widetilde{X}} g\left(\widetilde{X}_{0}\right)=\mathbb{k}$ and $D_{\widetilde{X}} g\left(\widetilde{X}_{0}\right)=(0, P)_{\mathbb{k} \times n}$, where $\operatorname{det} P_{\mathbb{k} \times \mathbb{k}} \neq 0$, then to the system (A.1)the following parametrisation can be applied:

$$
\begin{gathered}
\tilde{X}(t)=\psi(Y(t))=\widetilde{X}_{0}+U_{0} Y(t)+V_{0} h(Y(t)), \\
g(\psi(Y(t)))=0
\end{gathered}
$$

where

$$
\begin{aligned}
& U_{0}=\left(\begin{array}{c}
I_{(n-\mathbb{k})} \\
0
\end{array}\right)_{n \times(n-\mathbb{k})}, \quad V_{0}=\left(\begin{array}{c}
0 \\
I_{\mathbb{k}}
\end{array}\right)_{n \times \mathbb{k}}, \\
& Y(t)=\left(y_{1}(t), y_{2}(t), \cdots, y_{(n-\mathbb{k})}(t)\right)^{T} \in \mathrm{R}^{(n-\mathbb{k})},
\end{aligned}
$$

and $h(Y): \mathrm{R}^{(n-\mathbb{k})} \rightarrow \mathrm{R}^{\mathbb{k}}$ is a smooth mapping.

Substituting $\widetilde{X}(t)=\psi(Y(t))$ into the first equation (A.1) yields

$$
D_{Y} \psi(Y(t)) \dot{Y}(t)=f(\psi(Y(t)))
$$

Differentiating (A.2) in $Y$, we obtain

$$
D_{Y} \psi(Y(t))=U_{0}+V_{0} D_{Y} h(Y(t))
$$

and multiplying the result by $U_{0}^{T}$ leads to the equation

$$
U_{0}^{T} D_{Y} \psi(Y(t))=I_{(n-\mathbb{k})} .
$$

Now we differentiate the Eq. (A.3) in $Y$, so that

$$
D_{\widetilde{X}} g(\tilde{X}(t)) D_{Y} \psi(Y(t))=0 .
$$

It follows from (A.5) and (A.6) that

$$
D_{Y} \psi(Y(t))=\left(\begin{array}{c}
D_{\tilde{X}} g(\tilde{X}(t)) \\
U_{0}^{T}
\end{array}\right)^{-1}\left(\begin{array}{c}
0 \\
I_{(n-\mathbb{k})}
\end{array}\right),
$$

and substituting it into (A.4) yields

$$
\left(\begin{array}{c}
D_{\tilde{X}} g(\tilde{X}(t)) \\
U_{0}^{T}
\end{array}\right)^{-1}\left(\begin{array}{c}
0 \\
I_{(n-\mathbb{k})}
\end{array}\right) \dot{Y}(t)=f(\psi(Y(t))) .
$$

Using (A.4), (A.6) and (A.7), we write

$$
\left(\begin{array}{c}
0 \\
I_{(n-\mathbb{k})}
\end{array}\right) \dot{Y}(t)=\left(\begin{array}{c}
D_{\widetilde{X}} g(\tilde{X}(t)) f(\psi(Y(t))) \\
U_{0}^{T} f(\psi(Y(t)))
\end{array}\right)=\left(\begin{array}{c}
0 \\
U_{0}^{T} f(\psi(Y(t)))
\end{array}\right) .
$$


Thus the DAE system (A.1) can be reduced to the following parameterised system of $n-\mathbb{k}$ differential equations around its equilibrium $\widetilde{X}_{0}$ :

$$
\dot{Y}(t)=U_{0}^{T} f(\psi(Y(t)))
$$

the Taylor expansions of which around the equilibrium $\widetilde{X}_{0}$ has the form

$$
\dot{Y}=U_{0}^{T} D_{\widetilde{X}} f\left(\widetilde{X}_{0}\right)\left(\begin{array}{c}
D_{\widetilde{X}} g\left(\widetilde{X}_{0}\right) \\
U_{0}^{T}
\end{array}\right)^{-1}\left(\begin{array}{c}
0 \\
I_{(n-\mathbb{k})}
\end{array}\right) Y+o(|Y|) .
$$

\section{References}

[1] S. Ayasun, C.O. Nwankpa and H.G. Kwatny, Computation of singular and singularity induced bifurcation points of differential-algebraic power system model, IEEE Trans. Circuits Syst. I. Regul. Pap. 51, 1525-1537 (2004).

[2] S.M. Baer, B.W. Kooi, Y.A. Kuznetsov and H.R. Thieme, Multiparametric bifurcation analysis of a basic two-stage population model, SIAM J. Appl. Math. 66, 1339-1365 (2006).

[3] W.M. Boothby, An Introduction to Differential Manifolds and Riemannian Geometry, Academic Press (1986).

[4] K.E. Brenan, S.L. Campbell and L.R. Petzold, Numerical Solution of Initial-Value Problems in Differential-Algebraic Equations, SIAM (1996).

[5] B.S. Chen and J.J. Chen, Bifurcation and chaotic behavior of a discrete singular biological economic system, Appl. Math. Comput. 219, 2371-2386 (2012).

[6] B.S. Chen and J.J. Chen, Complex dynamic behaviors of a discrete predator-prey model with stage-structure and harvesting, Int. J. Biomath. 10, 1750013 (2017).

[7] B.S. Chen, X.X. Liao and Y.Q. Liu, Normal forms and bifurcations for the differential-algebraic systems (in Chinese), Acta Math. Appl. Sin. 23, 429-443 (2000).

[8] G.Y. Chen, Z.D. Teng and Z.Y. Hu, Analysis of stability for a discrete ratio-dependent predatorprey system, Indian J. Pure Appl. Math. 42, 1-26 (2011).

[9] L.S. Chen, Mathematical Models and Methods in Ecology (in Chinese), Science Press (2017).

[10] K.L. Cooke and Z. Grossman, Discrete delay, distributed delay and stability switches, J. Math. Anal. Appl. 86, 592-627 (1982).

[11] J.M. Cushing, An Introduction to Structured Population Dynamics, SIAM (1998).

[12] M. Fan and K. Wang, Harvesting problem with price changed with demand and supply (in Chinese), J. Biomath. 16, 411-415 (2001).

[13] T. Faria and L.T. Maglhalães, Normal form for retarded functional differential equations with parameters and applications to Hopf Bifurcation, J. Differential Equations 122, 181-200 (1995).

[14] K. Gapalsamy, Stability and Oscillations in Delay Equations of Population Dynamics, Kluwer Academic Publisher (1992).

[15] H.S. Gordon, Economic theory of a common property resource: the fishery, J. Polit. Econ. 62, 124-142 (1954).

[16] J. Guckenheimer and P. Holmes, Nonlinear Oscillations, Dynamical Systems, and Bifurcations of Vector Fields, Springer (1983).

[17] J. Hale and S.V. Lunel, Introduction to Functional Differential Equations, Springer (1993).

[18] B. Hassard, D. Kazarinoff and Y. Wan, Theory and Applications of Hopf Bifurcation, Cambridge University Press (1981).

[19] C.S. Holling, The components of predation as revealed by a study of small mammal predation of the European pine sawfly, Can. Entomol. 91, 293-320 (1959). 
Bifurcation in a Differential-Algebra Predator-Prey System with Time Lag Effects

[20] C.S. Holling, Some characteristics of simple types of predation and parasitism, Can. Entomol. 91, 385-398 (1959).

[21] A. Ilchmann and T. Reis (Eds.), Surveys in Differential-Algebraic Equations I, Springer (2013).

[22] A. Ilchmann and T. Reis (Eds.), Surveys in Differential-Algebraic Equations II-III, Springer (2015).

[23] A. Ilchmann and T. Reis (Eds.), Surveys in Differential-Algebraic Equations IV, Springer (2017).

[24] T.K. Kar and U.K. Pahari, Non-selective harvesting in prey-predator models with delay, Commun. Nonlinear Sci. Numer. Simul. 11, 499-509 (2006).

[25] M. Kot, Elements of Mathematical Biology, Cambridge University Press (2001).

[26] Y. Kuang, Delay Differential Equations with Application in Population Dynamics, Academic Press (1993).

[27] B. Leard, C. Lewis and J. Rebaza, Dynamics of ratio-dependent predator-prey models with nonconstant harvesting, Discrete Contin. Dyn. Syst. Ser. S 1, 303-315 (2008).

[28] M. Li, B.S. Chen and H.W. Ye, A bioeconomic differential algebraic predator-prey model with nonlinear prey harvesting, Appl. Math. Model. 42, 17-28 (2017).

[29] W. Liu and Y.L. Jiang, Dynamics of a modified predator-prey system to allow for a functional response and time delay, East Asian J. Appl. Math. 6, 384-399 (2016).

[30] W. Liu and Y.L. Jiang, Nonlinear dynamical behaviour in a predator-prey model with harvesting, East Asian J. Appl. Math. 7, 376-395 (2017).

[31] N.G. Mankiw, Principles of Economics, Peking University Press (2015).

[32] W. Marszalek and Z.W. Trzaska, Singularity-induced bifurcations in electrical power system, IEEE Trans. Power Syst. 20, 302-310 (2005).

[33] R.M. May, Time delay versus stability in population models with two and three trophic levels, Ecology 4, 315-325 (1973).

[34] L. Petzold, Differential/Algebraic Equations are not ODE's, SIAM J. Sci. Comput. 3, 367-384 (1982).

[35] S. Ruan and J. Wei, On the zeros of transcendental functions with applications to stability of delay differential equations with two delays, Dyn. Contin. Discrete Impuls. Syst. Ser. A Math. Anal. 10, 863-874 (2003).

[36] P.D.N. Srinivasu, S. Ismail and Ch.R. Naidu, Global dynamics and controllability of a harvested prey-predator system, J. Biol. Systems 9, 67-79 (2001).

[37] H.R. Thieme, Mathematics in Population Biology, Princeton University Press (2003).

[38] V. Venkatasubramanian, H. Schättler and J. Zaborszky, Local bifurcation and feasibility regions in differential-algebraic systems, IEEE Trans. Automat. Control 40, 1992-2013 (1995).

[39] X.Y. Wu and B.S. Chen, Bifurcations and stability of a discrete singular bioeconomic system, Nonlinear Dyn. 73, 1813-1828 (2013).

[40] Y.X. Xu and M.Y. Huang, Homoclinic orbits and Hopf bifurcations in delay differential systems with T-B singularity, J. Differential Equations 244, 582-598 (2008).

[41] K. Yosida, Functional Analysis, Springer (1995).

[42] Z.G. Zeng, P. Yu and X.X. Liao, A new comparison method for stability theory of differential systems with time-varying delays, Internat. J. Bifur. Chaos Appl. Sci. Engrg. 18, 169-186 (2008).

[43] Z.G. Zeng and W.X. Zheng, Multistability of neural networks with time-varying delays and concave-convex characteristics, IEEE Trans. Neural Netw. Learn. Syst. 23, 293-305 (2012).

[44] J.Y. Zhang and B.Y. Feng, Geometry Theory and Bifurcation Problems of Ordinary Differential Equations (in Chinese), Peking University Press (2000).

[45] G.D. Zhang and Y. Shen, Periodic solutions for a neutral delay Hassell-Varley type predator-prey system, Appl. Math. Comput. 264, 443-452 (2015).

[46] G.D. Zhang, Y. Shen and B.S. Chen, Positive periodic solutions in a non-selective harvesting 
predator-prey model with multiple delays, J. Math. Anal. Appl. 395, 298-306 (2012).

[47] G.D. Zhang, Y. Shen and B.S. Chen, Hopf bifurcation of a predator-prey system with predator harvesting and two delays, Nonlinear Dyn. 73, 2119-2131 (2013).

[48] G.D. Zhang, Y. Shen and B.S. Chen, Bifurcation analysis in a discrete differential-algebraic predator-prey system, Appl. Math. Model. 38, 4835-4848 (2014).

[49] G.D. Zhang and Z.G. Zeng, Exponential stability for a class of memristive neural networks with mixed time-varying delays, Appl. Math. Comput. 321, 544-554 (2018).

[50] G.D. Zhang, Z.G. Zeng and J.H. Hu, New results on global exponential dissipativity analysis of memristive inertial neural networks with distributed time-varying delays, Neural Netw. 97, 183-191 (2018). 